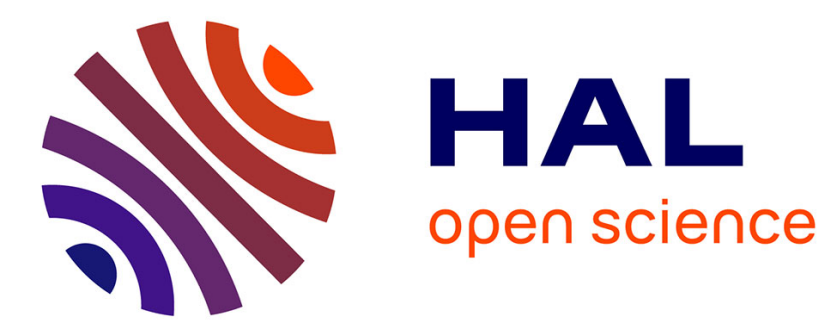

\title{
Enriched finite elements and local rescaling for vibrations of axially inhomogeneous Timoshenko beams
}

Rémi Cornaggia, Eric Darrigrand, Loïc Le Marrec, Fabrice Mahé

\section{To cite this version:}

Rémi Cornaggia, Eric Darrigrand, Loïc Le Marrec, Fabrice Mahé. Enriched finite elements and local rescaling for vibrations of axially inhomogeneous Timoshenko beams. Journal of Sound and Vibration, 2020, 474 (115228), 10.1016/j.jsv.2020.115228 . hal-02050532v2

\section{HAL Id: hal-02050532 \\ https://hal.science/hal-02050532v2}

Submitted on 4 Feb 2020

HAL is a multi-disciplinary open access archive for the deposit and dissemination of scientific research documents, whether they are published or not. The documents may come from teaching and research institutions in France or abroad, or from public or private research centers.
L'archive ouverte pluridisciplinaire HAL, est destinée au dépôt et à la diffusion de documents scientifiques de niveau recherche, publiés ou non, émanant des établissements d'enseignement et de recherche français ou étrangers, des laboratoires publics ou privés. 


\title{
Enriched finite elements and local rescaling for vibrations of axially inhomogeneous Timoshenko beams
}

\author{
R. Cornaggia a,*, E. Darrigrand ${ }^{\mathrm{b}}$, L. Le Marrec ${ }^{\mathrm{b}}$, F. Mahé ${ }^{\mathrm{b}}$ \\ ${ }^{a}$ Aix Marseille Univ, CNRS, Centrale Marseille, LMA UMR 7031, Marseille, France \\ ${ }^{b}$ Université de Rennes, CNRS, IRMAR - UMR 6625, F-35000 Rennes, France
}

\begin{abstract}
This work presents a new enriched finite element method dedicated to the vibrations of axially inhomogeneous Timoshenko beams. This method relies on the "half-hat" partition of unity and on an enrichment by solutions of the Timoshenko system corresponding to simple beams with a homogeneous or an exponentially-varying geometry. Moreover, the efficiency of the enrichment is considerably increased by introducing a new formulation based on a local rescaling of the Timoshenko problem, that accounts for the inhomogeneity of the beam. Validations using analytical solutions and comparisons with the classical high-order polynomial FEM, conduced for several inhomogeneous beams, show the efficiency of this approach in the time-harmonic domain. In particular low error levels are obtained over ranges of frequencies varying from a factor of one to thirty using fixed coarse meshes. Possible extensions to the research of natural frequencies of beams and to simulations of transient wave propagation are highlighted.
\end{abstract}

Keywords: Vibrations; Inhomogeneous Timoshenko beams; Enriched finite elements

\section{Introduction}

The Timoshenko model is widely used to describe the bending of beams, especially for vibration or wave propagation problems, as it approaches the dispersion relations of realistic 3D beams much more accurately than the simpler Rayleigh or Euler-Bernoulli models for medium and high frequencies [1, 2]. Numerous analytical and numerical methods based on this model have therefore been designed to address these problems, see $e . g$. the review by Hajianmaleki and Qatu [3].

Many of these works focus on homogeneous beams, but attention has also been dedicated to various geometrically or materially heterogeneous beams. Beams whose cross-section varies along the axial direction were studied from the 90 ' [4, 5, 6, 7], and this direction of research has been recently revived by the interest on periodically varying structures and the dynamic features (dispersion, band-gaps ...) they induce [8,9]. Moreover, the interest in composite materials, exhibiting continuously varying effective material parameters at the macroscopic level, lead to numerous studies of the so-called functionally graded beams (FGBs). These works propose several ways to take into account a material heterogeneity in the transverse direction [10], in the axial direction [7, 11], or in both directions [12]. Ultimately, beams that are both geometrically and materially heterogeneous are also considered [13, 14, 15].

Contrarily to the case of homogeneous beams, closed-form solutions of the Timoshenko system for these inhomogeneous beams are only available for simple exponential or polynomial variations of the geometric or material parameters [16, 11, 17, 18]. On the other hand, reliable and fast methods, accounting for the inhomogeneity, are needed for applications that require many computations, notably uncertainty quantification [19, 20] and optimization of shape or material mixture of inhomogeneous beams [7, 21], e.g. to enhance exotic properties such as the acoustic black-hole effect [22].

\footnotetext{
*Corresponding author.

Email addresses: cornaggia@lma.cnrs-mrs.fr (R. Cornaggia), eric.darrigrand-lacarrieu@univ-rennes1.fr (E. Darrigrand), loic.lemarrec@univ-rennes1.fr (L. Le Marrec), fabrice.mahe@univ-rennes1.fr (F. Mahé)
} 
To help meet this need, the present paper proposes a fast enriched finite element method (FEM) to compute the motion of geometrically heterogeneous beams submitted to time-harmonic loadings. This method is expected to provide a basis for eigenfrequency search [23], modal analysis of beams [24], and time-domain simulations by Fourier synthesis [2, 10, 15, 25].

The traditional FEM [26, 27] featuring low-order polynomial shape functions (also called $h$-FEM) is a reliable choice for static or low-frequency problems and slowly varying heterogeneities, but fails at addressing higher frequencies or sharper heterogeneities [27, 28]. To tackle these difficulties, two improvements are often considered: (i) using higher-order polynomial shape functions, the resulting method being sometimes called the $h p$-FEM [27, 29] or spectral FEM [30, 28], or (ii) enriching the finite element basis with problem-related functions chosen to approximate the exact solution better than polynomials. This second approach is adopted in this paper.

More specifically, the partition of unity method (PUM) by Melenk and Babuška [31] and its descendant the generalized FEM [32, 33] are considered. The PUM and related methods have already been applied to Timoshenko beams, e.g. to get rid of the "locking effect" that occur for very thin beams when using traditional FEM, for static loadings [34, 35, 36], vibrations [37] or transient motion [38]. In these studies, the enrichment functions are often chosen either as particular solutions of the static Timoshenko system for homogeneous beams, even when the focus is on the vibrations of heterogeneous beams as in [6, 13], or as oscillating functions whose design relies on numerical considerations rather than physical ones [37].

Based on these works, an efficient PUM is developed to address the vibrations of inhomogeneous Timoshenko beams. The proposed approach lies on two main components: new enrichment functions, and a new formulation of the Timoshenko problem.

On the one hand, new families of enrichment functions, which tackle both the heterogeneity of the beam and the time-harmonic nature of the motion are introduced. They are built developing a procedure introduced in a preliminary work [39] that addresses Webster's equation. This procedure features (i) a flexible "half-hat" partition of unity, and (ii) enrichment functions chosen as the solutions of the time-harmonic Timoshenko system for homogeneous or exponentially varying beams. Its implementation is identical to the one of the classical ninth-order polynomial FEM and therefore it can be incorporated in existing codes with reasonable implementation effort, and the static condensation procedure [27, 39] can be applied.

On the other hand, an original local rescaling, i.e. a position-dependent change of unknowns, is proposed to obtain a new formulation of the Timoshenko problem. This new formulation can also be discretized using the same enriched FEM. It partially accounts for the heterogeneity effects, thus the new unknowns are less affected by the heterogeneity, and therefore are much better approximated by the chosen enrichment functions.

The resulting method is first validated using the analytical solutions available for homogeneous and exponentially varying beams, and for boundary and distributed loadings. To show the efficiency of the method to handle the inhomogeneity of the beam, several numerical experiments and comparisons with a reference ninth-order polynomial FEM are then conduced on four beams with increasing complexity of inhomogeneity. Results are obtained in the following four configurations: (i) standard ninth-order polynomial FEM (ii) enriched FEM, (iii) local rescaling and polynomial FEM and (iv) local rescaling and enriched FEM. They assert the efficiency of combining the two proposed ingredients (configuration (iv)), especially for medium and high frequencies for which low error levels can still be obtained with very coarse meshes.

The paper is organized as follows. Section 2 presents the time-harmonic Timoshenko problem at hand, and gathers notations and existing results used throughout the paper. Section 3 briefly recalls the features of the FEM for Timoshenko beams and describes the enrichment procedure. The local rescaling and the associated modified formulation are introduced in Section 4. The numerical validations and examples are then presented in Section 5 . Finally, the application of the method to eigenfrequency search and time-domain simulations is discussed in Section 6, and the main results of the article are summarized in Section 7 .

\section{Notations and available exact solutions to the Timoshenko system}

Throughout the paper, a beam of length $\bar{L}$ is considered. It is submitted to in-plane time-harmonic loading at circular frequency $\bar{\omega}$, i.e. forces and momentums whose time dependency, omitted hereafter, is $\mathrm{e}^{-\mathrm{i} \bar{\omega} \bar{t}}$. Following the Timoshenko theory [1], the resulting in-plane motion is described by the time-harmonic transverse displacement of 


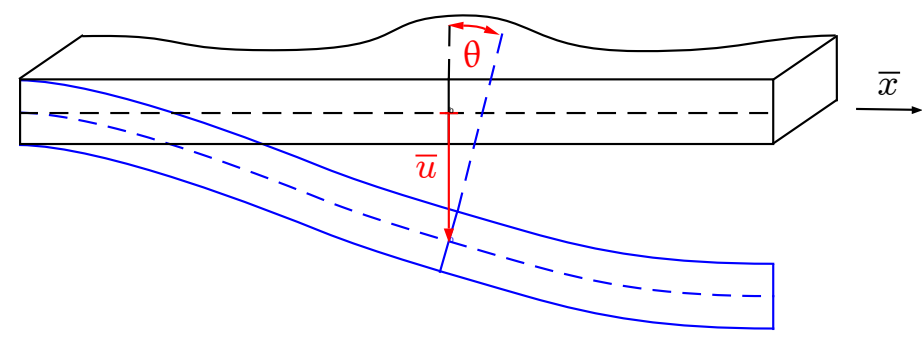

Figure 1: Bending of a geometrically heterogeneous beam: notation.

the mean axis (taken here as the $\bar{x}$-axis) and the rotation of the cross-section (Figure 1), whose amplitudes $\bar{u}$ and $\theta$ are solutions of the system:

$$
\left\{\begin{array}{r}
\frac{\mathrm{d}}{\mathrm{d} \bar{x}}\left(\kappa G A(\bar{x})\left(\frac{\mathrm{d} \bar{u}(\bar{x})}{\mathrm{d} \bar{x}}-\theta(\bar{x})\right)\right)+\rho A(\bar{x}) \bar{\omega}^{2} \bar{u}(\bar{x})+\bar{q}(\bar{x})=0 \\
\frac{\mathrm{d}}{\mathrm{d} \bar{x}}\left(E I(\bar{x}) \frac{\mathrm{d} \theta(\bar{x})}{\mathrm{d} \bar{x}}\right)+\kappa G A(\bar{x})\left(\frac{\mathrm{d} \bar{u}(\bar{x})}{\mathrm{d} \bar{x}}-\theta(\bar{x})\right)+\rho I(\bar{x}) \bar{\omega}^{2} \theta(\bar{x})+\bar{m}(\bar{x})=0
\end{array}\right.
$$

where $\rho, G$ and $E$ are respectively the density, the shear modulus and Young's modulus of the constitutive material of the beam; $A$ and $I$ are the area and quadratic momentum of the cross-section; $\kappa$ is the shear correction factor, which depends on the shape of the cross-section [40, 29]; and $\bar{q}$ and $\bar{m}$ are the spatial amplitudes of time-harmonic linear densities of forces and moments, respectively. In these equations, the shear force $\bar{N}$ and the bending moment $\bar{M}$ have been expressed in terms of the kinematic variables $(\bar{u}, \theta)$ thanks to the classical linear elastic constitutive relations $\bar{N}=\kappa G A(\mathrm{~d} \bar{u} / \mathrm{d} \bar{x}-\theta)$ and $\bar{M}=E I(\mathrm{~d} \theta / \mathrm{d} \bar{x})$.

Since the focus is on geometrically heterogeneous beams made of a homogeneous constitutive material, the geometrical parameters $A$ and $I$ depend on $\bar{x}$, while the material parameters $\rho, G$ and $E$ are uniform. To simplify ensuing computations, it is additionally assumed that the shape of the cross-section varies sufficiently slowly so that the correction factor $\kappa$ can also be considered uniform.

To complete the system (1), two boundary conditions (BCs) must be imposed at each extremity of the beam, each pair of conditions corresponding to a kind of support. Classical examples of these supports include the glued extremity $(\bar{u}=0$ and $\theta=0)$; the simply supported extremity $(\bar{u}=0$ and $\bar{M}=0)$; the vertical roller connection $(\bar{N}=0$ and $\theta=0)$; and the free extremity $(\bar{N}=0$ and $\bar{M}=0)$.

\subsection{Dimensionless Timoshenko system}

For further convenience, the system (1) is now reformulated into a dimensionless form, similarly to e.g. [41, 42]. To this end, constant characteristic amplitudes $A_{\mathrm{c}}$ and $I_{\mathrm{c}}$ are introduced. These will be chosen as $A_{\mathrm{c}}=A(0)$ and $I_{\mathrm{c}}=I(0)$ thereafter, but other choices are possible, e.g. $A_{\mathrm{c}}$ and $I_{\mathrm{c}}$ may be the mean values of $A$ and $I$ along the beam. Then, the relevant scales of the problem are the gyration radius $r_{\mathrm{c}}$, Timoshenko's cut-off frequency $\omega_{\mathrm{c}}$, and the shear-compression ratio $g$ defined by:

$$
r_{\mathrm{c}}=\sqrt{\frac{I_{\mathrm{c}}}{A_{\mathrm{c}}}}, \quad \omega_{\mathrm{c}}=\frac{1}{r_{\mathrm{c}}} \sqrt{\frac{G}{\rho}}, \quad g=\frac{E}{\kappa G} .
$$

In particular, for most materials, one has $2 \lesssim g \lesssim 3$. Dimensionless counterparts of the coordinate, parameters, fields and unknowns that appear in the system (1) are defined thanks to these scales as:

$$
x=\frac{\bar{x}}{r_{\mathrm{c}}}, \quad L=\frac{\bar{L}}{r_{\mathrm{c}}}, \quad u=\frac{\bar{u}}{r_{\mathrm{c}}}, \quad \omega=\frac{\bar{\omega}}{\omega_{\mathrm{c}}}, \quad q=\frac{\bar{q} r_{\mathrm{c}}}{\kappa G A_{\mathrm{c}}} \quad \text { and } \quad m=\frac{\bar{m} r_{\mathrm{c}}^{2}}{\kappa G I_{\mathrm{c}}} .
$$

The dimensionless and strictly positive functions $\mathcal{A}$ and $\mathcal{I}$, that are called profiles of the area and quadratic momentum hereafter, are similarly defined as:

$$
\mathcal{A}(x)=\frac{A(\bar{x})}{A_{\mathrm{c}}} \quad \text { and } \quad \mathcal{I}(x)=\frac{I(\bar{x})}{I_{\mathrm{c}}} .
$$


Combining the definitions (3) and (4) with the original system (1), one founds that the couple of dimensionless unknowns $(u, \theta)$ satisfies:

$$
\left\{\begin{aligned}
\left(\mathcal{A}\left(u^{\prime}-\theta\right)\right)^{\prime}+\omega^{2} \mathcal{A} u+q & =0 \\
g\left(\mathcal{I} \theta^{\prime}\right)^{\prime}+\mathcal{A}\left(u^{\prime}-\theta\right)+\omega^{2} \mathcal{I} \theta+m & =0
\end{aligned}\right.
$$

where' stands for differentiation with respect to the dimensionless coordinate $x$. By analogy with the system (1), the dimensionless shear force $N$ and bending moment $M$ are given by:

$$
N=\mathcal{A}\left(u^{\prime}-\theta\right) \quad \text { and } \quad M=g \mathcal{I} \theta^{\prime},
$$

so that $N(x)=\bar{N}(\bar{x}) / \kappa G A_{\mathrm{c}}$ and $M(x)=r_{\mathrm{c}} \bar{M}(\bar{x}) / \kappa G I_{\mathrm{c}}$. To complete the problem, boundary conditions must be applied on the couples $(u, \theta),(u, M),(N, \theta)$ or $(N, M)$ at both extremities, $x=0$ and $x=L$.

For the reader's convenience, Table 1 summarizes the notations introduced above.

\begin{tabular}{|l||c|c|}
\hline Parameters & Physical & Dimensionless \\
\hline \hline Longitudinal coordinate and total beam length & $\bar{x}$ and $\bar{L}$ & $x$ and $L$ \\
\hline Material parameters: Young's modulus, shear modulus and density & $E, G$ and $\rho$ & - \\
\hline Geometrical parameters: area and quadratic momentum & $A$ and $I$ & $\mathcal{A}$ and $\mathcal{I}$ \\
\hline Correction factor & $\kappa$ & - \\
\hline Shear-compression ratio & - & $g$ \\
\hline Circular frequency of the time-harmonic loads and motion & $\bar{\omega}$ & $\omega$ \\
\hline Amplitudes of the applied linear force and moment & $\bar{q}$ and $\bar{m}$ & $q$ and $m$ \\
\hline Amplitudes of the displacement and rotation of the mean axis & $\bar{u}$ and $\theta$ & $u$ and $\theta$ \\
\hline Amplitudes of the internal shear force and bending moment & $\bar{N}$ and $\bar{M}$ & $N$ and $M$ \\
\hline
\end{tabular}

Table 1: Notations for the physical quantities (parameters, fields etc.) that intervene in the problem and their dimensionless counterparts. The symbol "-" means that the associated parameter is not defined.

Remark 1. In the system (5) and afterwards, the dimensionless circular frequency $\omega$ can equivalently be replaced by a dimensionless "reference wavenumber" $k_{0}$. To do so, one first defines a physical reference wavenumber $\overline{k_{0}}=$ $\bar{\omega} / \sqrt{G / \rho}$, whose dimensionless counterpart is $k_{0}=r_{c} \overline{k_{0}}=\omega$. The notation $\omega$ was retained $(i)$ to stress the importance of the time-harmonic assumption throughout the article and (ii) so that the dispersion relations and wavenumbers given in the next section are written in terms of the usual notations.

Remark 2. The Timoshenko system can often be reduced to only one fourth-order equation. For geometrically heterogeneous beams and when no linear density of forces is applied $(q=0)$, it was pointed out by Huang et al. [14] that the displacement $u$ and rotation $\theta$ may be both written in terms of a new unknown function $f$ as:

$$
u=\frac{f^{\prime}}{\mathcal{A}}, \quad \theta=\left(\frac{f^{\prime}}{\mathcal{A}}\right)^{\prime}+\omega^{2} \frac{f}{\mathcal{A}}
$$

Then the shear force is $N=\mathcal{A}\left(u^{\prime}-\theta\right)=-\omega^{2} f$ and the transverse equilibrium equation $N^{\prime}+\omega^{2} \mathcal{A} u=0$ is automatically verified. The rotation equilibrium equation becomes a fourth-order equation in $f$ :

$$
\left(\mathcal{I}\left[\left(\frac{f^{\prime}}{\mathcal{A}}\right)^{\prime}+\omega^{2} \frac{f}{\mathcal{A}}\right]^{\prime}\right)^{\prime}+\omega^{2}\left(\mathcal{I}\left[\left(\frac{f^{\prime}}{\mathcal{A}}\right)^{\prime}+\omega^{2} \frac{f}{\mathcal{A}}\right]-f\right)+m=0 .
$$

However, the classical system (5) is preferred hereafter, as (i) it enables to consider nonzero density of forces $q$ (ii) the boundary conditions involving $\theta$ or $M$ are more easily expressed and (iii) the corresponding weak formulation of the problem (see Section 3.1) is posed in the usual functional space $H^{1}(0, L)$, whereas a weak formulation obtained from the fourth-order equation (8) would be posed in $H^{2}(0, L)$ and the choice of a finite element basis would therefore be more constrained. 


\subsection{Available exact solutions for free vibrations}

Some exact solutions available in the literature for the free vibrations of Timoshenko beams, i.e. solutions of the system (5) with $q=0$ and $m=0$, are now recalled. These solutions will be used to build enriched finite element spaces in the next section, and to validate the whole method in Section 5

Homogeneous beams. Homogeneous beams are addressed in many textbooks e.g. [1]. In this case, the profiles $\mathcal{A}$ and $\mathcal{I}$ are uniform and the only remaining geometrical parameter of the problem is the constant $\alpha:=\mathcal{A} / \mathcal{I}$. The system (5) becomes:

$$
\left\{\begin{aligned}
\left(u^{\prime}-\theta\right)^{\prime}+\omega^{2} u & =0 \\
g \theta^{\prime \prime}+\alpha\left(u^{\prime}-\theta\right)+\omega^{2} \theta & =0
\end{aligned}\right.
$$

Looking for oscillating solutions with the same wavenumber $k$ for $u$ and $\theta$, i.e. $u(x)=u_{0} \mathrm{e}^{\mathrm{i} k x}$ and $\theta(x)=\theta_{0} \mathrm{e}^{\mathrm{i} k x}$, one obtains the dispersion relation:

$$
g K^{2}-\omega^{2}(g+1) K+\omega^{2}\left(\omega^{2}-\alpha\right)=0, \quad \text { with } \quad K:=k^{2},
$$

which admits two real solutions:

$$
K_{ \pm}=\frac{\omega^{2}(g+1) \pm \omega \sqrt{4 g \alpha+\omega^{2}(g-1)^{2}}}{2 g} .
$$

The four values that can be taken by the wavenumber $k$ are therefore:

$$
k_{1}=\sqrt{K_{+}}, \quad k_{2}=-\sqrt{K_{+}}, \quad k_{3}=\sqrt{K_{-}}, \quad k_{4}=-\sqrt{K_{-}},
$$

These wavenumbers are represented in Figure 2 as $\omega$ increases. Note in particular that $K_{+}>0$, so that $k_{1}$ and $k_{2}$ are real, whereas $K_{-}$has the same sign as $\omega-\sqrt{\alpha}$, so that $k_{3}$ and $k_{4}$ are imaginary at low frequencies [2]. For high frequencies, one has $K_{+} \sim \omega^{2}$ and $K_{-} \sim \omega^{2} / g$ as $\omega \rightarrow \infty$, regardless of the value of $\alpha$ (Figure 2).

Finally, the basis of solutions for the Timoshenko system 9 , parametrized by $\alpha$, is:

$$
\Psi^{\omega, \alpha}=\left\{x \mapsto \mathrm{e}^{\mathrm{i} k_{m} x}\right\}_{m=1 \ldots 4} .
$$

Remark 3. If the beam is homogeneous on the whole domain $[0, L]$, by choosing $A_{\mathrm{c}}=A$ and $I_{\mathrm{c}}=I$ in the scaling (4), one obtains $\mathcal{A}=\mathcal{I}=1$ and $\alpha=1$. For piecewise homogeneous beams, if the same scaling is used throughout the whole beam (by e.g. choosing $A_{\mathrm{c}}$ and $I_{\mathrm{c}}$ as the mean values of $A$ and I), $\alpha$ takes different values in each subdomain and different bases of solutions $\Psi^{\omega, \alpha}$ should be used throughout the beam.

Exponentially varying beams. Closed-form solutions also exist for beams whose profiles are exponentially varying at the same rate: $\mathcal{A}(x)=\mathcal{A}_{0} \mathrm{e}^{2 \delta x}$ and $\mathcal{I}(x)=\mathcal{I}_{0} \mathrm{e}^{2 \delta x}$, e.g. beams with rectangular cross-sections having a constant thickness and an exponentially varying width [17, 18]. Keeping the notation $\alpha=\mathcal{A} / \mathcal{I}=\mathcal{A}_{0} / \mathcal{I}_{0}$, the system (5) becomes:

$$
\left\{\begin{aligned}
\left(u^{\prime}-\theta\right)^{\prime}+2 \delta\left(u^{\prime}-\theta\right)+\omega^{2} u & =0 \\
g\left(\theta^{\prime \prime}+2 \delta \theta^{\prime}\right)+\alpha\left(u^{\prime}-\theta\right)+\omega^{2} \theta & =0 .
\end{aligned}\right.
$$

Looking for oscillating solutions $\mathrm{e}^{\mathrm{i} k x}$ for both $u$ and $\theta$, the dispersion relation is found to be:

$$
g \widehat{K}^{2}-\omega^{2}(1+g) \widehat{K}+\omega^{2}\left(\omega^{2}-\alpha\right)=0, \text { with } \widehat{K}=k^{2}-2 \mathrm{i} \delta k .
$$

This is exactly the equation (10) obtained for homogeneous beams, except that $K=k^{2}$ is replaced by $\widehat{K}$. The roots are $K_{+}$and $K_{-}$, as given by $[11$, , and the associated wavenumbers are:

$$
\widehat{k}_{1}=\mathrm{i} \delta+\sqrt{K_{+}-\delta^{2}}, \quad \widehat{k}_{2}=\mathrm{i} \delta-\sqrt{K_{+}-\delta^{2}}, \quad \widehat{k}_{3}=\mathrm{i} \delta+\sqrt{K_{-}-\delta^{2}}, \quad \widehat{k}_{4}=\mathrm{i} \delta-\sqrt{K_{-}-\delta^{2}} .
$$

These wavenumber are also represented in Figure 2 for completeness. Eventually, the basis of solutions of the system (14), parametrized by $\alpha$ and $\delta$, is:

$$
\Psi^{\omega, \alpha, \delta}=\left\{x \mapsto \mathrm{e}^{\mathrm{i} \widehat{\mathrm{k}}_{m} x}\right\}_{m=1 \ldots 4}=\left\{x \mapsto \mathrm{e}^{-\delta x} \mathrm{e}^{\mathrm{i} s_{m} x} \sqrt{\left(k_{m}\right)^{2}-\delta^{2}}\right\}_{m=1 \ldots 4}, \quad s_{m}:=(-1)^{m-1} .
$$



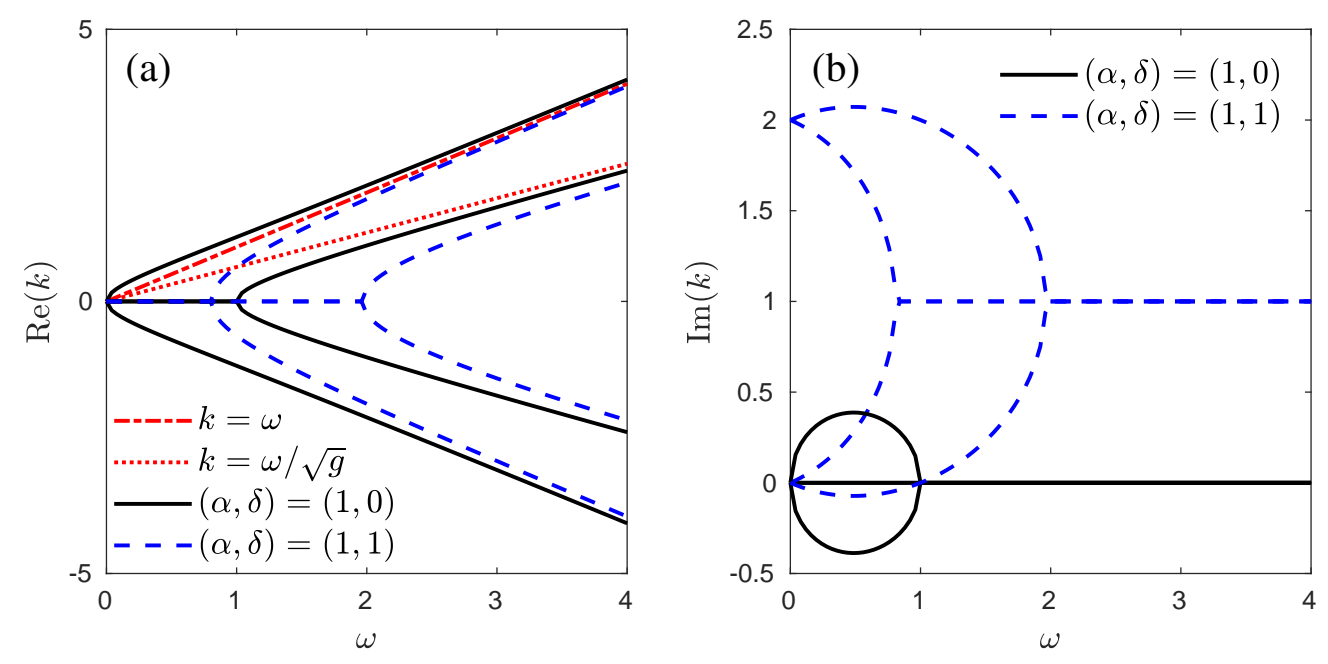

Figure 2: Examples of dispersion relations for homogeneous and exponentially varying beams: (a) real and (b) imaginary parts of the wavenumbers $k_{m}(\omega)$ (solid) and $\widehat{k}_{m}(\omega)$ (dashed) defined by [12) and (16). The shear-compression ratio is $g=2.5$.

Other exact solutions. The paper by Yuan et al. [18] provides other closed-form solutions, for several "complex exponential" (i.e. exponential of polynomials) and polynomial profiles. These solutions are written in terms of hypergeometric functions. Exact solutions are also built by Sarkar and Ganguli [11] for geometrically homogeneous but materially graded beams. An inverse point of view is adopted: a polynomial solution $(u, \theta)$ that satisfies prescribed boundary conditions is chosen, and then one determines the polynomial profiles of material parameters that permit to recover this solution. Semi-analytical solutions are also available, e.g. Eisenberger [16] considers polynomial variations of $\mathcal{A}$ and $\mathcal{I}$ and expands the solution as a power series, whose coefficients are shown to satisfy recurrence relations, and Sohani and Eipakchi [42] propose a perturbation approach based on the WKB expansion to tackle the heterogeneity.

\section{Enriched finite elements}

In this section, the principles of the Finite Element Method (FEM) applied to the Timoshenko system are briefly recalled (see e.g. the textbooks [27, 26] for further details). An enrichment method, that produces approximation spaces dedicated to specific problems, is then presented. Spaces dedicated to time-harmonic Timoshenko problems are finally built thanks to this method.

\subsection{Weak formulation}

The first step to apply the FEM is to write the weak formulation of the problem. First, some notations for the functional spaces used thereafter are recalled [26, App. B]. As usual, $L^{2}(0, L)$ denotes the space of square-integrable functions on $] 0, L[$ and:

$$
\begin{aligned}
H^{1}(0, L) & :=\left\{f \text { such that } f \in L^{2}(0, L) \text { and } f^{\prime} \in L^{2}(0, L)\right\}, \\
H_{(0}^{1}(0, L) & :=\left\{f \in H^{1}(0, L), f(0)=0\right\}, \\
H_{0}^{1}(0, L) & :=\left\{f \in H^{1}(0, L), f(0)=0 \text { and } f(L)=0\right\} .
\end{aligned}
$$

Multiplying the two lines of the system (5) by two test functions $v$ and $\phi$, integrating by parts, summing the resulting equalities, one obtains the weak formulation of the Timoshenko problem (i.e. of the system (5) associated 
with a given set of $\mathrm{BCs}$ ) as:

$$
\begin{aligned}
& \text { Find }(u, \theta) \in \mathcal{V} \text { such that } \begin{aligned}
\int_{0}^{L}\left[\mathcal{A}\left(u^{\prime}-\theta\right)\left(v^{\prime}-\phi\right)+g \mathcal{I} \theta^{\prime} \phi^{\prime}-\omega^{2}(\mathcal{A} u v+\mathcal{I} \theta \phi)\right] \mathrm{d} x \\
\qquad=[N v+M \phi]_{0}^{L}+\int_{0}^{L}(q v+m \phi) \mathrm{d} x \quad \text { for all }(v, \phi) \in \mathcal{V}_{0},
\end{aligned}
\end{aligned}
$$

where the space of kinematically admissible fields $\mathcal{V}$ is a subspace of $\left(H^{1}(0, L)\right)^{2}$ that depends on the kinematic boundary conditions that are imposed on $u$ or $\theta ; \mathcal{V}_{0}$ is the associated linear space (corresponding to homogeneous kinematic BCs); and the boundary term $[N v+M \phi]_{0}^{L}$ depends on all BCs. Some examples include:

- the glued-glued beam, for which $(u, \theta)$ vanishes at both extremities. In this case, $\mathcal{V}=\mathcal{V}_{0}=\left(H_{0}^{1}(0, L)\right)^{2}$, the boundary term vanishes and the motion is only due to the force density $q$ or the moment density $m$.

- the cantilever beam, for which $(u, \theta)(0)=(0,0)$ and the shear force and bending moment are imposed at the right extremity: $N(L)=N_{\star}$ and $M(L)=M_{\star}$. In this case, the functional space is $\mathcal{V}=\mathcal{V}_{0}=\left(H_{(0}^{1}(0, L)\right)^{2}$, and the boundary contribution is:

$$
[N v+M \phi]_{0}^{L}=N_{\star} v(L)+M_{\star} \phi(L)
$$

- the glued-free beam submitted to time-harmonic displacement with amplitude $u_{\star}$ at $x=0$ (i.e. $u(0)=u_{\star}$ and $\theta(0)=0)$ and free at $x=L($ i.e. $N(L)=0$ and $M(L)=0)$. Then one has:

$$
\mathcal{V}=\left\{(u, \theta) \in H^{1}(0, L) \times H_{(0}^{1}(0, L), u(0)=u_{\star}\right\}, \quad \mathcal{V}_{0}=\left(H_{(0}^{1}(0, L)\right)^{2},
$$

and the boundary term entirely vanishes.

In the third example above, and more generally when non-homogeneous kinematic BCs are imposed, $\mathcal{V}$ is an affine space, i.e. $\boldsymbol{V} \neq \boldsymbol{V}_{0}$. However, one easily comes back to the configuration $\boldsymbol{V}=\boldsymbol{V}_{0}$ by considering a lifting of a problem, i.e. by defining new unknowns $\left(u_{\ell}, \theta_{\ell}\right):=(u, \theta)-(\hat{u}, \hat{\theta}) \in \mathcal{V}_{0}$, where $(\hat{u}, \hat{\theta})$ is an arbitrary element of $\mathcal{V}$.

For instance, taking $(\hat{u}, \hat{\theta})=\left(\hat{u}_{\star}, 0\right)$, where $\hat{u}_{\star}$ is a chosen function of $H^{1}(0, L)$ such that $\hat{u}_{\star}(0)=u_{\star}$, the lifted counterpart of the glued-free beam problem (19)-(21) is:

$$
\begin{aligned}
& \text { Find }\left(u_{\ell}, \theta_{\ell}\right) \in \mathcal{V}_{0} \text { such that } \int_{0}^{L}\left[\mathcal{A}\left(u_{\ell}^{\prime}-\theta_{\ell}\right)\left(v^{\prime}-\phi\right)+g \mathcal{I} \theta_{\ell}^{\prime} \phi^{\prime}-\omega^{2}\left(\mathcal{A} u_{\ell} v+\mathcal{I} \theta_{\ell} \phi\right)\right] \mathrm{d} x \\
& =\int_{0}^{L}(q v+m \phi) \mathrm{d} x-\int_{0}^{L}\left[\mathcal{A}\left(\hat{u}_{\star}^{\prime}\left(v^{\prime}-\phi\right)-\omega^{2} \hat{u}_{\star} v\right)\right] \mathrm{d} x \quad \text { for all }(v, \phi) \in \mathcal{V}_{0},
\end{aligned}
$$

where the space $\boldsymbol{V}_{0}$ is given by (21). Then the solution of the original problem is $(u, \theta)=\left(u_{\ell}+\hat{u}_{\star}, \theta_{\ell}\right)$.

Since such a lifting is always possible, it is assumed that $\boldsymbol{V}=\boldsymbol{V}_{0}$ in all the considered problems hereafter.

\subsection{Finite element method}

The finite element method [27, 26] belongs to the family of Galerkin methods, that rely on an approximation of the trial space $\boldsymbol{V}_{0}$ by a finite-dimension subspace $\boldsymbol{V}_{h, 0} \subset \mathcal{V}_{0}$. In this work, the same approximation space is used for $u$ and $\theta$. As a consequence, one first looks for a subspace $\mathcal{V}_{h}$ of $H^{1}(0, L)$, whose dimension is noted $N_{h}$, and then defines $\boldsymbol{V}_{h}=\left(\mathcal{V}_{h}\right)^{2}$ the corresponding space of couples $\left(u_{h}, \theta_{h}\right)$. The subspace $\boldsymbol{V}_{h, 0}=\boldsymbol{V}_{0} \cap \boldsymbol{V}_{h}$ is finally built by removing the functions that does not satisfy the kinematic boundary conditions on $u$ or $\theta$ from the basis of $\mathcal{V}_{h}$.

In practice, this last step is performed at the end of the discretization process described now. First, the displacement and the rotation angle are approximated using the same basis $\left\{\varphi_{j}\right\}_{j=1 \ldots N_{h}}$ of $\mathcal{V}_{h}$, i.e. one looks for approximations $\left(u_{h}, \theta_{h}\right)$ of $(u, \theta)$ as:

$$
u_{h}=\sum_{j=1}^{N_{h}} u_{j} \varphi_{j} \quad \text { and } \quad \theta_{h}=\sum_{j=1}^{N_{h}} \theta_{j} \varphi_{j}
$$


By inserting these approximations into the weak formulation $(19)$, and using the couples $(v, \phi)=\left(\varphi_{i}, 0\right)$ and $(v, \phi)=$ $\left(0, \varphi_{i}\right)$ as test functions, one obtains the $2 N_{h} \times 2 N_{h}$ linear system:

$$
\left(\boldsymbol{K}-\omega^{2} \boldsymbol{M}\right) \cdot \boldsymbol{U}=\boldsymbol{F},
$$

where the vector $\boldsymbol{U}$ contains the discrete unknowns $u_{j}$ and $\theta_{j}$. In this work, these values are disposed alternatively:

$$
\boldsymbol{U}=\left[u_{1}, \theta_{1}, \ldots, u_{j}, \theta_{j}, \ldots u_{N_{h}} \theta_{N_{h}}\right]^{\mathrm{T}}, \quad \text { i.e. } \quad U_{2 j-1}=u_{j} \quad \text { and } \quad U_{2 j}=\theta_{j}, \quad j=1 \ldots N_{h},
$$

so that the components of the stiffness matrix $\boldsymbol{K}$ are:

$$
\begin{array}{rlrl}
K_{2 i-1,2 j-1} & =\int_{0}^{L} \mathcal{A} \varphi_{i}^{\prime} \varphi_{j}^{\prime} \mathrm{d} x, \quad K_{2 i-1,2 j} & =-\int_{0}^{L} \mathcal{A} \varphi_{i}^{\prime} \varphi_{j} \mathrm{~d} x, \\
K_{2 i, 2 j-1} & =-\int_{0}^{L} \mathcal{A} \varphi_{i} \varphi_{j}^{\prime} \mathrm{d} x, \quad K_{2 i, 2 j}=\int_{0}^{L}\left[\mathcal{A} \varphi_{i} \varphi_{j}+g \mathcal{I} \varphi_{i}^{\prime} \varphi_{j}^{\prime}\right] \mathrm{d} x,
\end{array}
$$

and those of the mass matrix $\boldsymbol{M}$ are:

$$
M_{2 i-1,2 j-1}=\int_{0}^{L} \mathcal{A} \varphi_{i} \varphi_{j} \mathrm{~d} x, \quad M_{2 i, 2 j}=\int_{0}^{L} \mathcal{I} \varphi_{i} \varphi_{j} \mathrm{~d} x,
$$

and $M_{2 i-1,2 j}=M_{2 i, 2 j-1}=0$. The right-hand-side vector $\boldsymbol{F}$ is decomposed into $\boldsymbol{F}=\boldsymbol{F}^{\text {in }}+\boldsymbol{F}^{\mathrm{bc}}$, where $\boldsymbol{F}^{\text {in }}$ contains the contribution of the force and moment densities:

$$
F_{2 i-1}^{\mathrm{in}}=\int_{0}^{L} q \varphi_{i} \mathrm{~d} x, \quad F_{2 i}^{\mathrm{in}}=\int_{0}^{L} m \varphi_{i} \mathrm{~d} x,
$$

and $\boldsymbol{F}^{\mathrm{bc}}$ accounts for the boundary conditions on $N$ or $M$. For instance, for glued-glued beams one has $\boldsymbol{F}^{\mathrm{bc}}=\mathbf{0}$, and for cantilever beams, using 20 one has $\boldsymbol{F}^{\mathrm{bc}}=\boldsymbol{F}^{\mathrm{bc}, \mathrm{ct}}$ with:

$$
F_{2 i-1}^{\mathrm{bc}, \mathrm{ct}}=N_{\star} \varphi_{i}(L), \quad F_{2 i}^{\mathrm{bc}, \mathrm{ct}}=M_{\star} \varphi_{i}(L) .
$$

The steps described above only take into account the boundary conditions on $N$ and $M$. The last step to adapt the system (24) to a given problem is to impose that $u_{h}$ and $\theta_{h}$ satisfy the prescribed kinematic BCs. To this end, the functions $\varphi_{j}$ that does not satisfy these BC are removed from the basis (equivalently, the associated coefficients $u_{j}$ and $\theta_{j}$ are set to 0 in (23)). The corresponding lines and columns are finally removed from the system (24).

The quality of the approximation obtained by solving this system depends on the interpolation properties of the chosen space $\mathcal{V}_{h}$, i.e. the minimal distance between the exact solution of the system and a function of $\boldsymbol{V}_{h}$. For many applications, well-documented spaces of piecewise-polynomial functions [27, 26] offer very good performances. However, for some problems, including medium- or high-frequency vibration problems, polynomial FE need refined meshes and therefore high computational cost to catch the fast oscillations of the solution. The proposed alternative is to build enriched spaces by incorporating oscillating functions into the elementary bases, as presented now.

\subsection{Building enriched spaces using the "half-hat" partition of unity}

Enrichment functions are incorporated in an approximation space following the partition of unity method (PUM) introduced by Melenk and Babuška [31], which is adapted to the Timoshenko problem as follows. One must first define a partition of unity, i.e. a family of functions $\left\{\varphi_{n}\right\}_{n=0 . . N_{\mathrm{e}}}$ that satisfies:

$$
\sum_{n=0}^{N_{\mathrm{e}}} \varphi_{n}(x)=1, \quad \forall x \in[0, L] .
$$

Given another family of functions $\Psi=\left\{\psi^{m}\right\}_{m=1 \ldots N_{m}}$ that gathers functions that will be added in the approximation space, and that is called the enrichment family hereafter, one then builds an enriched function space $\mathcal{V}_{h}^{\Psi}$ as:

$$
\mathcal{V}_{h}^{\Psi}=\operatorname{span}\left\{\varphi_{n}^{m}\right\}_{n=0 . . N_{\mathrm{e}}, m=0 . N_{m}} \quad \text { with }\left\{\begin{array}{l}
\varphi_{n}^{0}=\varphi_{n}, \\
\varphi_{n}^{m}=\varphi_{n} \psi^{m} .
\end{array}\right.
$$


(a)

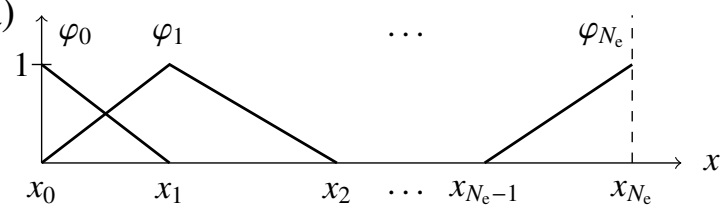

(b)

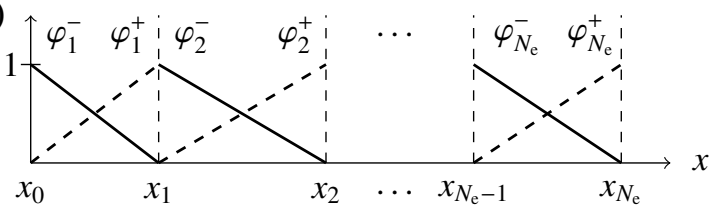

Figure 3: (a) "Hat" and (b) "half-hat" functions defined from a mesh $\left\{x_{0}, x_{1}, \ldots, x_{N_{\mathrm{e}}}\right\}$.

A function $u_{h} \in \mathcal{V}_{h}^{\Psi}$ is defined by its $\left(N_{\mathrm{e}}+1\right) \times\left(N_{m}+1\right)$ components $u_{n}^{m}$ in the basis $\left\{\varphi_{n}^{m}\right\}$ :

$$
u_{h}=\sum_{n=0}^{N_{\mathrm{e}}} \sum_{m=0}^{N_{m}} u_{n}^{m} \varphi_{n}^{m}
$$

The most commonly used PU is the family of "hat" functions, to which the notation $\varphi_{n}$ will apply hereafter, defined from a mesh $\left\{0=x_{0}, x_{1}, \ldots, x_{N_{\mathrm{e}}}=L\right\}$ of $[0, L]$ as plotted in Figure 3 . Indeed, these functions form a basis of the traditional FE space $\mathcal{P}_{1}$ of piecewise-linear functions [26], and some convenient properties of this basis are then transmitted the enriched space, notably the sparsity of stiffness and mass matrices [31, 32].

However, with such a PU the additional functions $\varphi_{n}^{m}=\varphi_{n} \psi^{m}$ are supported by two elements. To restrain their support to one element only, it was proposed in a previous work [39] to use the "half-hat" PU represented in Figure 3 instead. Since on the $e$-th element the couple $\left\{\varphi_{\mathrm{e}}^{-}, \varphi_{\mathrm{e}}^{+}\right\}$forms a local partition of unity, one can work directly on the elementary basis and choose a specific enrichment family $\Psi_{e}$ for each element. The additional functions should also cancel at the end of each element to simplify the implementation of the resulting method and to be able to apply the static condensation procedure, as specified in [39] and in Section 5.1 below.

For each function $\psi_{\mathrm{e}}^{m}$ in the enrichment family $\Psi_{e}$, two additional functions $\varphi_{\mathrm{e}}^{m-}$ and $\varphi_{\mathrm{e}}^{m+}$ are therefore added to the elementary basis. Written as functions of the normalized coordinate $\xi=\left(x-x_{e-1}\right) / h_{e} \in[0,1]$, where $h_{e}:=x_{e}-x_{e-1}$ is the length of the $e$-th element, they read:

$$
\varphi_{\mathrm{e}}^{m-}(\xi)=(1-\xi)\left[\psi_{\mathrm{e}}^{m}\left(x_{e-1}+h_{e} \xi\right)-\psi_{\mathrm{e}}^{m}\left(x_{e-1}\right)\right], \quad \varphi_{\mathrm{e}}^{m+}(\xi)=\xi\left[\psi_{\mathrm{e}}^{m}\left(x_{e-1}+h_{e} \xi\right)-\psi_{\mathrm{e}}^{m}\left(x_{e}\right)\right]
$$

Remark 4. When the space generated by the enrichment family is invariant by shifting the origin of the x-axis (typically, when the family embeds only exponential functions e.g. $\psi_{\mathrm{e}}^{m}(x)=\exp \left(\mathrm{i} k_{m} x\right)$ ), it is convenient to use the definitions:

$$
\varphi_{\mathrm{e}}^{m-}(\xi)=(1-\xi)\left[\psi_{\mathrm{e}}^{m}\left(h_{e} \xi\right)-\psi_{\mathrm{e}}^{m}(0)\right], \quad \varphi_{\mathrm{e}}^{m+}(\xi)=\xi\left[\psi_{\mathrm{e}}^{m}\left(h_{e}(\xi-1)\right)-\psi_{\mathrm{e}}^{m}(0)\right],
$$

instead of (32), in particular to simplify the implementation. These alternative definitions are used hereafter.

Then, approximations $\left(u_{h}, \theta_{h}\right)$ are sought as linear combinations of (i) "hat" functions, to ensure the continuity of these approximations [39], and (ii) those inner additional functions. Hereafter, each element contains the same number $N_{m}$ of enrichment functions, and such a combination is therefore written:

$$
u_{h}=\sum_{n=0}^{N_{\mathrm{e}}} u_{n}^{0} \varphi_{n}^{0}+\sum_{e=1}^{N_{\mathrm{e}}} \sum_{m=1}^{N_{m}}\left[u_{e}^{m+} \varphi_{\mathrm{e}}^{m+}+u_{\mathrm{e}}^{m-} \varphi_{\mathrm{e}}^{m-}\right]
$$

In the sum (34), the distinction is made between the nodal values $u_{n}^{0}$ associated with the "hat" functions, and the inner values $u_{\mathrm{e}}^{m \pm}$ associated with the additional inner functions. The approximation space embedding all these combinations is:

$$
\mathcal{P}_{1,2 m}=\operatorname{span}\left\{\left\{\varphi_{n}^{0}\right\}_{n=0 . N_{\mathrm{e}}} \bigcup\left\{\varphi_{e}^{m+}, \varphi_{\mathrm{e}}^{m-}\right\}_{e=1 \ldots N_{\mathrm{e}}, m=1 \ldots N_{m}}\right\},
$$

whose dimension is $\operatorname{dim} \mathcal{P}_{1,2 m}=\left(N_{\mathrm{e}}+1\right)+2 N_{\mathrm{e}} \times N_{m}$.

Finally, specifying kinematic boundary conditions corresponding to a given problem is done by fixing only the nodal boundary values $u_{0}^{0}=u_{h}(0)$ and $u_{N_{\mathrm{e}}}^{0}=u_{h}(L)$, and similarly for $\theta_{h}$, while boundary conditions on the shear force $N$ and moment $M$ are accounted for in the weak formulation (19). 
Remark 5. The notation was slightly modified compared to the previous work [39]: the "half-hat" functions $\varphi_{\mathrm{e}}^{ \pm}$and elementary functions $\varphi_{\mathrm{e}}^{m \pm}$ are now associated with a given element (thus the subscript ${ }^{\circ}$ ); and the + and - exponents now indicate the sign of the slope of the functions $\varphi_{\mathrm{e}}^{ \pm}$.

\subsection{Enriched spaces dedicated to Timoshenko beams}

To conclude this section, approximation spaces are built by combining (i) the bases of solutions for homogeneous or exponential beams given in Section (2.2) and (ii) the enrichment procedure described above. Spaces that will be used as reference in the numerical illustrations are also presented.

Timoshenko-enriched spaces. A first enriched space, noted $\mathcal{P}_{1,8}^{\omega, \alpha}$, is built from the functions of the family $\Psi^{\omega, \alpha}$ given by (13) and that describe the free vibrations of a homogeneous beam. For an arbitrary heterogeneous beam, the function $\alpha=\mathcal{A} / \mathcal{I}$ is used to define enrichements in each elementary basis: the $e$-th basis is enriched with the family $\Psi^{\omega, \alpha_{e}}$, where $\alpha_{e}:=\alpha\left(\left(x_{e-1}+x_{e}\right) / 2\right)$ is the value of $\alpha$ at the middle point of the $e$-th element. Following [33), the eight additional inner functions of the basis are:

$$
\varphi_{\mathrm{e}}^{m-}(\xi)=(1-\xi)\left[\mathrm{e}^{\mathrm{i} k_{m} h_{e} \xi}-1\right] \quad \text { and } \quad \varphi_{\mathrm{e}}^{m+}(\xi)=\xi\left[\mathrm{e}^{\mathrm{i} k_{m} h_{e}(\xi-1)}-1\right] \quad(m=1 \ldots 4),
$$

where the wavenumbers $k_{m}$ given by (12) depend on $\alpha_{e}$. These functions are represented in Figure 4(c). The enriched space then embeds the exact solution for a piecewise-homogeneous or "step" beam, free from distributed loading.

The space $\mathcal{P}_{1, \delta}^{\omega, \alpha, \delta}$ is introduced next. Its elementary bases are enriched with the functions of the family $\Psi^{\omega, \alpha_{e}, \delta_{e}}$ given by (17) and that describe the free vibrations of exponential beams. For each element, the parameters $\alpha_{e}$ and $\delta_{e}$ are the values of the functions $\alpha$ and $\delta:=\mathcal{A}^{\prime} / 2 \mathcal{A}$, evaluated at the middle of the element. The additional inner functions are defined similarly to $\left[36\right.$, but with the wavenumbers $\left\{k_{m}\right\}_{m=1 \ldots 4}$ replaced by their counterparts $\left\{\widehat{k}_{m}\right\}_{m=1 \ldots 4}$ defined by (16).

In the particular case of beams with constant thickness, $\alpha=\mathcal{A} / \mathcal{I}$ is constant and therefore all elementary bases are identical for the space $\mathcal{P}_{1,8}^{\omega, \alpha}$ : it is then a globally enriched space that does not accounts for the inhomogeneity of the beam. In this case, using the second space $\mathcal{P}_{1,8}^{\omega, \alpha, \delta}$, which is still locally enriched when $\delta$ is inhomogeneous, is a first way to take the width variations into account.

Remark 6. The spaces $\mathcal{P}_{1,8}^{\omega, \alpha}$ and $\mathcal{P}_{1,8}^{\omega, \alpha, \delta}$ are the counterparts for Timoshenko beams of the spaces $\mathcal{P}_{1,4}^{k}$ and $\mathcal{P}_{1,4}^{k, \delta}$ built in [39] from time-harmonic solutions corresponding to homogeneous and exponentially varying bars.

For completeness, some other enriched spaces that produced similar results on the upcoming test-cases are presented in Appendix A.

Reference spaces. To assert the efficiency of the enriched spaces, their performances will be compared to those of more classical spaces. The first reference that comes to mind is the simplest finite element method that uses the "hat" functions as elementary basis, i.e. the approximation space is the space $\mathcal{P}_{1}$ of piecewise linear functions. However, this space is well-known to perform poorly to tackle medium or high-frequency problems, especially compared to higher-order polynomial spaces, see e.g. [27, Sect. 4.7] and [28] for theoretical and numerical comparisons.

On the basis of the analysis of the enrichment method [39], the enriched spaces built above are expected to perform much better than $\mathcal{P}_{1}$. In fact, since their elementary bases include eight enrichment functions, they are expected to share the convergence properties of the space $\mathcal{P}_{9}$ of piecewise ninth-order polynomials, at fixed frequency. To present a fair comparison, the space $\mathcal{P}_{9}$ is chosen as a reference rather than $\mathcal{P}_{1}$.

Several elementary bases exist for the space $\mathcal{P}_{9}$, among them are the hierarchical basis of Lobatto polynomials (often implicitly associated with the so-called $p$ or $h p$-FEM [27, Sect. 4.7]) and the "spectral" basis of Lagrange polynomials with interpolation nodes taken as the Gauss-Lobatto-Legendre points of the reference element [30, 28]. These two bases were compared by Sprague and Geers [28], and produced nearly identical results in terms of accuracy. Since the main advantage of spectral bases, namely the diagonal mass matrices they produce, is irrelevant for timeharmonic problems, a hierarchical basis, represented in Figure 4 (a), is chosen in this work for simplicity.

Finally, to complete the comparison, the performance of a "naively" sine-enriched space will also be explored. This space is enriched with the simplest family of oscillating functions $\Psi^{\omega}:=\left\{x \mapsto \mathrm{e}^{ \pm \mathrm{i} \omega x}\right\}$, as proposed by many other authors e.g. [37, 38]. To maintain eight inner functions as in the other considered bases, Lobatto polynomials 
up to the fifth degree are also added to obtain a polynomial-enriched space denoted by $\mathcal{P}_{5,4}^{\omega}$, whose elementary basis is represented in Figure 4 (b).

Remark 7. The implementation of the FEM is identical for both the reference and enriched spaces: all the elementary bases include two nodal and eight inner functions.
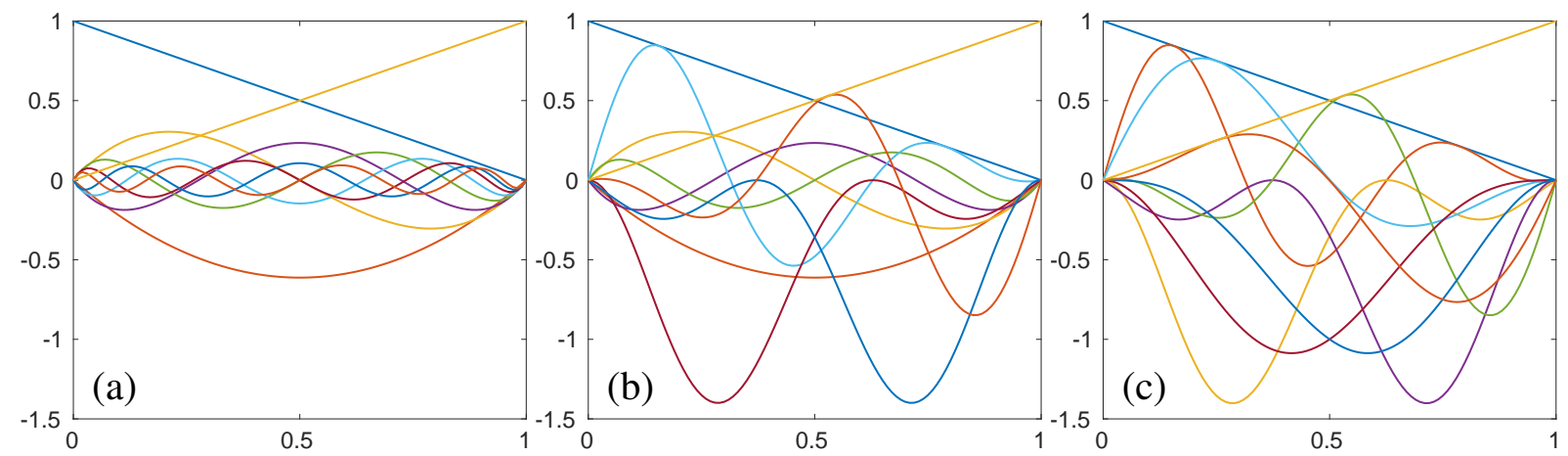

Figure 4: Elementary basis functions on an element of length $h=1$ : (a) hierarchical basis of Lobatto polynomials (space $\mathcal{P}_{9}$ ); (b) sine-enriched basis for $\omega=10$ ( $\operatorname{space} \mathcal{P}_{5,4}^{\omega}$ ); and (c) Timoshenko-enriched basis for $\omega=10$ and $\alpha=1$ (space $\mathcal{P}_{1,8}^{\omega, \alpha}$ ).

\section{A new formulation based on a local rescaling}

For arbitrarily varying profiles $\mathcal{A}, \mathcal{I}$, the enrichment functions corresponding to homogeneous or exponentially varying beams account only partially for the geometry. To improve again the efficiency of the enriched method, geometrical information is now incorporated into the problem unknowns before the discretization.

\subsection{Change of unknowns in the Timoshenko system}

First, the variable coefficients $(d, a)$ are defined as:

$$
d=\sqrt{\mathcal{A}} \text { and } a=\sqrt{\mathcal{I}},
$$

and new unknowns $(\tilde{u}, \tilde{\theta})$ are introduced thanks to the local, i.e. $x$-dependent, rescaling:

$$
u=\frac{\tilde{u}}{d}, \quad \theta=\frac{\tilde{\theta}}{a} .
$$

This change of unknowns is inspired by a similar rescaling for Webster's equation $\left(\mathcal{A} u^{\prime}\right)^{\prime}+k^{2} \mathcal{A} u+f=0$, which models the longitudinal motion of beams [39], or low-frequency acoustic propagation in waveguides [43]. Indeed, when the profile $\mathcal{A}$ is regular enough, Webster's equation can be converted into a Helmholtz equation with variable wavenumber:

$$
\left(\mathcal{A} u^{\prime}\right)^{\prime}+k^{2} \mathcal{A} u+f=0 \quad \Longleftrightarrow \quad \tilde{u}^{\prime \prime}+\left(k^{2}-\frac{d^{\prime \prime}}{d}\right) \tilde{u}+\frac{f}{d}=0
$$

with $u=\tilde{u} / d$ and $d=\sqrt{\mathcal{A}}$.

Similarly, introducing the rescaling [38) into the Timoshenko system (5) leads to:

$$
\left\{\begin{array}{r}
\left(d \tilde{u}^{\prime}-d^{\prime} \tilde{u}-\frac{d^{2}}{a} \tilde{\theta}\right)^{\prime}+\omega^{2} d \tilde{u}+q=0 \\
g\left(a \tilde{\theta}^{\prime}-a^{\prime} \tilde{\theta}\right)^{\prime}+\left(d \tilde{u}^{\prime}-d^{\prime} \tilde{u}-\frac{d^{2}}{a} \tilde{\theta}\right)+\omega^{2} a \tilde{\theta}+m=0 .
\end{array}\right.
$$


Equivalently, expanding (40) and dividing the two lines by $d$ and $a$, respectively, one obtains:

$$
\left\{\begin{aligned}
\tilde{u}^{\prime \prime}+\left(\omega^{2}-\frac{d^{\prime \prime}}{d}\right) \tilde{u} & =\frac{d a \tilde{\theta}^{\prime}+\left(2 d^{\prime} a-d a^{\prime}\right) \tilde{\theta}}{a^{2}}-\frac{q}{d} \\
g \tilde{\theta}^{\prime \prime}+\left(\omega^{2}-\frac{d^{2}}{a^{2}}-g \frac{a^{\prime \prime}}{a}\right) \tilde{\theta} & =-\frac{d \tilde{u}^{\prime}-d^{\prime} \tilde{u}}{a}-\frac{m}{a} .
\end{aligned}\right.
$$

The system (41) models two coupled harmonic oscillators, where the variable geometric parameters $d$ and $a$ intervene in the wavenumbers and the coupling terms, but not in the second-order terms in $\tilde{u}$ and $\tilde{\theta}$. The new unknowns $\tilde{u}$ and $\tilde{\theta}$ are expected to be less affected by the inhomogeneity than the original solutions $(u, \theta)$, and therefore better approximated by oscillating enrichment functions corresponding to homogeneous beams. Of note, this is the case for exponentially varying beams with $\mathcal{A}=\mathcal{I}$ : the solutions given by (17) are written $u=\tilde{u} / \sqrt{\mathcal{A}}$ where $\tilde{u}$ is an oscillating function with homogeneous amplitude.

\subsection{Discretization}

The weak formulation associated with the system 40 is:

$$
\begin{aligned}
& \text { Find }(\tilde{u}, \tilde{\theta}) \in \widetilde{\mathcal{V}} \text { such that } \begin{aligned}
\int_{0}^{L}\left[\left(d \tilde{u}^{\prime}-d^{\prime} \tilde{u}-\frac{d^{2}}{a} \tilde{\theta}\right)\left(v^{\prime}-\phi\right)+g\left(a \tilde{\theta}^{\prime}-a^{\prime} \tilde{\theta}\right) \phi^{\prime}-\omega^{2}(d u v+a \tilde{\theta} \phi)\right] \mathrm{d} x \\
=[N v+M \phi]_{0}^{L}+\int_{0}^{L}(q v+m \phi) \mathrm{d} x \quad \text { for all }(v, \phi) \in \mathcal{V}_{0},
\end{aligned}
\end{aligned}
$$

where $(v, \phi)$ still denote the test functions, and $\widetilde{\mathcal{V}}$ is obtained by modifying the non-homogeneous kinematic boundary conditions embedded in the definition of $\mathcal{V}$ accordingly with the change of unknowns $[38$. The associated linear space is $\widetilde{\boldsymbol{V}}_{0}=\boldsymbol{V}_{0}$, so that, up to a lifting, the problem (42) may be also written using only $\boldsymbol{V}_{0}$.

After discretization, the associated finite element system is:

$$
\left(\widetilde{\boldsymbol{K}}-\widetilde{\boldsymbol{D}}-\omega^{2} \widetilde{\boldsymbol{M}}\right) \cdot \widetilde{\boldsymbol{U}}=\boldsymbol{F}
$$

where the components of the stiffness and mass matrices $\widetilde{\boldsymbol{K}}$ and $\widetilde{\boldsymbol{M}}$ have similar expressions than those of $\boldsymbol{K}$ and $\boldsymbol{M}$ defined by 26 27, with $\mathcal{A}$ and $\mathcal{I}$ replaced respectively by $d$ and $a$, except for the components $\left\{\widetilde{K}_{2 i, 2 j}\right\}_{i, j=1 \ldots N_{h}}$ where $\mathcal{A}$ is replaced by $d^{2} / a$ instead. The matrix $\widetilde{\boldsymbol{D}}$ embeds the contributions of the derivatives $d^{\prime}$ and $a^{\prime}$ arising in (42) after the change of unknowns:

$$
\begin{aligned}
\widetilde{D}_{2 i-1,2 j-1} & =\int_{0}^{L} d^{\prime} \varphi_{i}^{\prime} \varphi_{j} \mathrm{~d} x, & \widetilde{D}_{2 i-1,2 j} & =-\int_{0}^{L} d^{\prime} \varphi_{i} \varphi_{j} \mathrm{~d} x, \\
\widetilde{D}_{2 i, 2 j-1} & =0, & \widetilde{D}_{2 i, 2 j} & =\int_{0}^{L} g a^{\prime} \varphi_{i}^{\prime} \varphi_{j} \mathrm{~d} x,
\end{aligned}
$$

and the right-hand side vector $\boldsymbol{F}$ is left unchanged and is defined by (29). Contrary to the system (24), the system (43) is not symmetric, due to the contribution of $\widetilde{\boldsymbol{D}}$.

\subsection{Enriched spaces}

Finally, approximations $\left(\tilde{u}_{h}, \tilde{\theta}_{h}\right)$ of $(\tilde{u}, \tilde{\theta})$ will be sought in the spaces $\mathcal{P}_{9}, \mathcal{P}_{5,4}^{\omega}$ and $\mathcal{P}_{1,8}^{\omega, \alpha}$ defined in Section 3.4 . The corresponding spaces of solutions $(u, \theta)$ embedding the local rescaling are denoted by $\widetilde{\mathcal{P}}$, e.g. :

$$
\widetilde{\mathcal{P}}_{9}:=\left\{\left(\frac{\tilde{u}_{h}}{d}, \frac{\tilde{\theta}_{h}}{a}\right),\left(\tilde{u}_{h}, \tilde{\theta}_{h}\right) \in\left(\mathcal{P}_{9}\right)^{2}\right\}, d=\sqrt{\mathcal{A}}, a=\sqrt{\mathcal{I}} .
$$

Enriched spaces $\widetilde{\mathcal{P}}_{5,4}^{\omega}$ and $\widetilde{\mathcal{P}}_{1,8}^{\omega, \alpha}$ are defined similarly: when $d$ and $a$ are constant, the system (40) degenerates into the $\alpha$-dependent system (9) with $\alpha=d^{2} / a^{2}$, so using the associated solutions is still relevant for the modified formulation. On the other hand, there is no physically-based counterpart to the space $\mathcal{P}_{1,8}^{\omega, \alpha, \delta}$ and attempts to define a similar enriched space were not conclusive, see Appendix A 


\section{Numerical validation and illustrations}

In this part, some general peculiarities of the implementation of the enriched FEM are first presented. Then, the validation of the method is exposed: enriched spaces are compared with the reference first- and ninth-order polynomial spaces on test-cases with analytical solutions. Finally, simulations of the vibrations of four beams with various heterogeneous geometries are presented and discussed.

All the tests are performed on cantilever beams, glued at their left extremity $x=0$, i.e. $(u, \theta)(0)=(0,0)$. In most of the studied configurations, the chosen loading is a time-harmonic transverse force with unit amplitude imposed at the right extremity $x=L$, i.e. one sets $(N, M)(L)=(1,0), q=0$ and $m=0$. In one occasion (Subsection 5.3.2), a nonzero time-harmonic density of force is chosen instead, with the right extremity left free, i.e. one sets $(N, M)(L)=(1,0)$, $q=q_{\mathrm{P}} \neq 0$ and $m=0$. The results were obtained with an implementation of the method in a MATLAB framework, and using a good laptop $(4 \times 2.60 \mathrm{GHz} \mathrm{CPU}$ and $32 \mathrm{Go} \mathrm{RAM})$.

\subsection{Implementation choices}

Numerical integration. To compute the matrices' components [26|27|44], a classical Gauss quadrature [26, Sect. 8] was chosen. In addition, the following procedure ensures that the integration of oscillatory basis functions remains accurate even for large elements supporting several wavelengths.

A reference dimensionless wavelength independent of the considered beam is first introduced:

$$
\lambda:=2 \pi / k_{0}=2 \pi / \omega,
$$

where $k_{0}$ is the reference wavenumber defined in Remark 1 . This reference wavelength is the smallest wavelength in homogeneous and exponentially varying beams at the high-frequency limit, as discussed in Section 2.2. Then, each element is divided into $N_{\mathrm{se}}:=\lceil h / 2 \lambda\rceil$ subelements of equal lengths, where $h=\max _{n=1 \ldots N_{\mathrm{e}}} h_{n}$ and $\lceil\cdot\rceil$ denotes the ceiling function, so that $\lceil x\rceil-1<x \leq\lceil x\rceil$ for any $x \in \mathbb{R}$. Then, 10 Legendre-Gauss points are used in each subelement to compute its contribution to the total integral. In this way, each subelement covers at most half of a wavelength $\lambda$, and there are at least 20 integration points per wavelength.

Static condensation. Since all the elementary bases presented above feature two nodal and eight inner basis functions, the same static condensation (SC) procedure can be applied to the linear systems (24) and (43). This procedure is described in details e.g. in the monograph by Ihlenburg [27, Sec. 4.7.3], and is also called dynamic reduction by some authors [25, Sect. 2.3]. In short, it first intervenes in the matrices assembly process: the inner values are expressed in terms of the nodal values by inverting each $8 \times 8$ inner elementary matrix. Then only the nodal values are retained as unknowns, and the global matrix to be inverted is therefore much smaller than the initial one, e.g. $2 N_{\mathrm{e}} \times 2 N_{\mathrm{e}}$ instead of $10 N_{\mathrm{e}} \times 10 N_{\mathrm{e}}$ for a cantilever beam. This matrix is also better conditioned than the original one, while only the small $8 \times 8$ elementary matrices inverted at the condensation step suffer from bad conditioning. Finally a post-processing operation (the decondensation) is needed to retrieve the inner values from the node values.

\subsection{Error and expected results for varying resolutions}

In the upcoming comparisons, the accuracy of a FE solution $\left(u_{h}, \theta_{h}\right)$ compared to the exact solution $(u, \theta)$ is measured by the relative error $E_{\mathcal{L}}$ defined by:

$$
E_{\mathcal{L}}\left(u_{h}, \theta_{h}\right):=\left\|\left(u_{h}-u, \theta_{h}-\theta\right)\right\|_{\mathcal{L}} /\|(u, \theta)\|_{\mathcal{L}}
$$

where the Lagrangian-based norm $\|\cdot\|_{\mathcal{L}}$ is:

$$
\|(u, \theta)\|_{\mathcal{L}}=(-\mathcal{L}(u, \theta))^{1 / 2}=\left(\int_{0}^{L}\left[\mathcal{A}\left(u^{\prime}-\theta\right)^{2}+g \mathcal{I}\left(\theta^{\prime}\right)^{2}+\omega^{2}\left(\mathcal{A} u^{2}+\mathcal{I} \theta^{2}\right)\right] \mathrm{d} x\right)^{1 / 2},
$$

and $\mathcal{L}(u, \theta)$ is the Lagrangian of the state $(u, \theta)$, i.e. the difference between the associated kinetic and elastic energies. This norm was also chosen for its similarity with the weighted $H^{1}$-norm $\|u\|_{H^{1}, k}^{2}:=\left\|u^{\prime}\right\|_{L^{2}}^{2}+k^{2}\|u\|_{L^{2}}^{2}$ that appears in the analysis of problems modeled by the Helmholtz equation $-u^{\prime \prime}-k^{2} u=f$, to balance the weight of a function $u$ and 
its derivatives for oscillating solutions. In practice, a discrete counterpart of the integral (48) is computed using the middle-point method and a very thin dedicated mesh (1000 elements) independent of the FE meshes.

In most of the upcoming examples, the accuracy of the FE solutions will be sought for several resolutions $\lambda / h$, where $h=\max _{n=1 \ldots N_{\mathrm{e}}} h_{n}$ is the largest element length and $\lambda=2 \pi / \omega$ is the reference wavelength. The resolution is an indicator of the number of elements per smallest wavelength, that is a relevant criterion to compare several FE spaces having the same number of basis functions per element when solving time-harmonic problems. The variation of the resolution is performed in two ways:

- First, the frequency $\omega$ is fixed and the error is computed for several regular meshes, i.e. for different element length $h$. For piecewise-smooth profiles $(\mathcal{A}, \mathcal{I})$, the exact solution is also piecewise-smooth. Therefore, using the ninth-order polynomial space $\mathcal{P}_{9}$, the error (47) should decrease as $O\left(h^{9}\right)$ as the mesh size $h$ decreases, according to the classical theory for polynomial finite elements [27, 26] and provided that nodes are placed at the singularities of the profiles or their derivatives. Based on previously proved results for similar enriched spaces [39], the errors obtained with the ninth-order enriched spaces $\left(\mathcal{P}_{5,4}^{\omega}, \mathcal{P}_{1,8}^{\omega, \alpha}, \ldots\right)$ are also expected to decrease with the same rate.

- Then, the mesh is fixed and the frequency $\omega$ increases. In this way, the frequency-dependent peculiarities of the enriched spaces are observed. Indeed, the accuracy obtained with polynomial spaces depends mainly on the resolution $\lambda / h$, with a pollution error increasing with the frequency [27, Sect. 4.6]. On the contrary, the accuracy of enriched spaces is expected to be more stable thanks to the oscillatory enrichment functions determined from the frequency of each problem.

\subsection{Validation of the method}

First, the implementation of the polynomial and enriched FE bases are validated by comparison with the analytical solutions recalled in Section 2.2 for homogeneous and exponential beams.

\subsubsection{Homogeneous beams}

For homogeneous beams, simulation results are omitted for brevity, but the following key properties have been verified:

- The Timoshenko-enriched space $\mathcal{P}_{1,8}^{\omega, \alpha}$, that embeds the analytical solution, produces errors close to machine precision when no distributed loading is applied $(q=0$ and $m=0)$.

- The other spaces $\left(\mathcal{P}_{9}\right.$ and $\left.\mathcal{P}_{5,4}^{\omega}\right)$ produce errors that decrease with the same ninth-order rate at fixed frequency (i.e. as $O\left(h^{9}\right)$ as $\left.h \rightarrow 0\right)$.

Moreover, the initial and modified formulations are identical, thus the effect of the local rescaling cannot be observed.

\subsubsection{Exponentially varying beams}

The tests performed on an exponentially varying beam of length $L=10$ are now presented in more details. The chosen profiles are:

$$
\mathcal{A}(x)=\mathcal{I}(x)=\mathrm{e}^{2 \delta x},
$$

with $\delta$ chosen such that $\mathcal{A}(L)=1 / 8$. For these profiles, the space $\mathcal{P}_{1,8}^{\omega, \alpha}$ does not contain the exact solution for free vibrations (17), but its "exponentially" enriched companion $\mathcal{P}_{1,8}^{\omega, \alpha, \delta}$ does. Moreover, the initial and modified formulations differ. Both the effects of the enrichments and of the modified formulation can therefore be observed.

Point-like loading. First, a cantilever beam whose motion is only due to a time-harmonic transverse force applied at its right extremity, with amplitude $N(L)=1$, is considered. In this case the exact solution is a combination of the free vibrations solutions given by (17).

To illustrate the convergence properties discussed in Section 3.4 , the three kinds of ninth-order spaces (polynomial space $\mathcal{P}_{9}$ and enriched spaces $\mathcal{P}_{5,4}^{\omega}$ and $\mathcal{P}_{1,8}^{\omega, \alpha}$ with respectively four and eight enrichment functions per elementary basis) are compared to the simplest first-order polynomial space $\mathcal{P}_{1}$. To this end, the relative errors obtained at the fixed frequency $\omega=5$ are plotted in Figure 5 versus the number of degrees of freedom $N_{\text {dof }}$. Nine times more elements 
are used for $\mathcal{P}_{1}$ than for the other spaces to obtain the same $N_{\text {dof }}$. As expected, all the ninth-order (polynomial and enriched) spaces produce the same ninth-order convergence rate in the convergence regime, and therefore completely outperform the first-order space that (i) enters the convergence regime later and (ii) converges much slower.

As a complementary illustration, Table 2 also provides a comparison of the meshes, numbers of degrees of freedom and CPU times needed to reach a relative error less than $0.1 \%$. The much larger number of elements needed with the first-order space results in a considerably larger computational time than with the ninth-order spaces, to reach a similar precision.

In the ensuing discussion, only polynomial and enriched ninth-order spaces are therefore compared. The effects of the modified formulation are studied. The resolution (number of elements per wavelength) is used rather than the number of degrees of freedom as indicator of the discretization. As the convergence order is always $O\left(h^{9}\right)$ for these spaces, the slopes indicators are omitted hereafter.

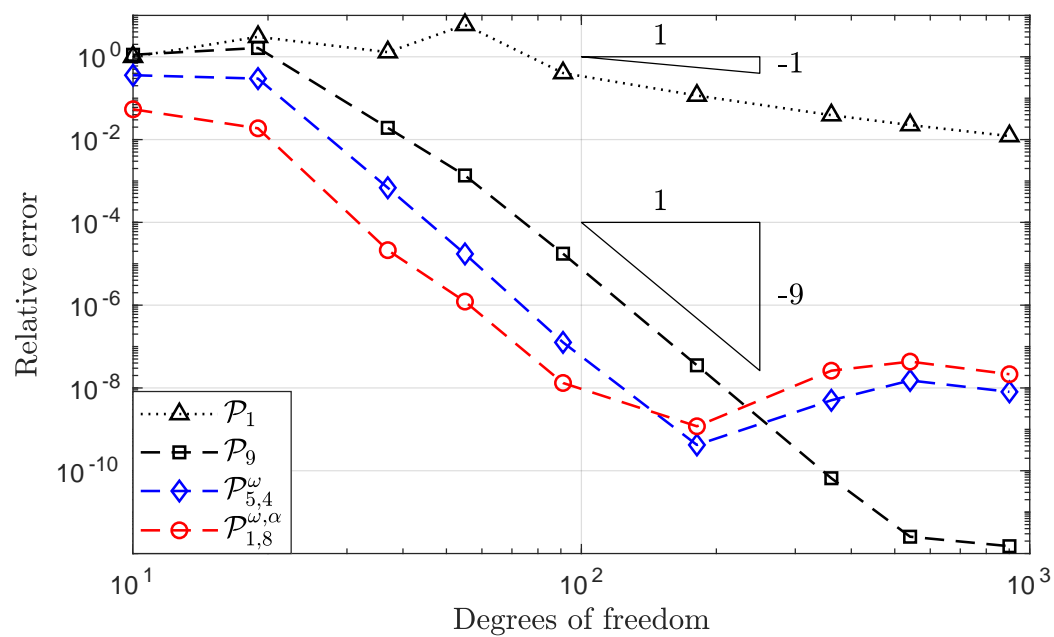

Figure 5: Relative errors for a cantilever beam with exponential profiles, with $g=2.5$ and at frequency $\omega=5$, versus the number of degrees of freedom, using a first-order polynomial space and ninth-order polynomial and enriched spaces. The triangles indicate the theoretical slopes in the convergence regime for first-order and ninth-order spaces.

\begin{tabular}{|c||c|c|c|c|}
\hline FE space & Error & $N_{\mathrm{e}}$ & $N_{\text {dof }}$ & CPU time \\
\hline $\mathcal{P}_{1}$ & $9.9 \times 10^{-4}$ & 10300 & 20600 & $11.6 \mathrm{~s}$ \\
\hline $\mathcal{P}_{9}$ & $3.7 \times 10^{-4}$ & 7 & 126 & $0.60 \mathrm{~s}$ \\
\hline $\mathcal{P}_{5,4}^{\omega}$ & $6.9 \times 10^{-4}$ & 4 & 72 & $0.42 \mathrm{~s}$ \\
\hline $\mathcal{P}_{1,8}^{\omega, \alpha}$ & $5.3 \times 10^{-4}$ & 3 & 54 & $0.47 \mathrm{~s}$ \\
\hline
\end{tabular}

Table 2: Meshes and computational times needed to reach a relative error less than $0.1 \%$ for an exponential cantilever beam, at $\omega=5$ (same configuration than in Figure 5. The CPU time includes the time taken by (i) the matrix assembly process, (ii) the resolution of the linear system and (iii) the computation of the approximate solution on the grid points used to compute the error. It is measured using the functions tic and toc of MATLAB and averaged over 10 realizations of the same process.

In Figure 6 are plotted the displacement and rotation fields $(u, \theta)$ obtained at frequency $\omega=5$ with a coarse mesh made of only $N_{\mathrm{e}}=2$ elements, so that the resolution is $\lambda / h \approx 1 / 4$ (four wavelengths per element). In this case, the sine-enriched space $\mathcal{P}_{5,4}^{\omega}$ outperforms the polynomial space $\mathcal{P}_{9}$ in the approximation of the displacement $u$ (i.e. replacing four polynomials by oscillating functions in the elementary basis already improves the quality of the results) and is outperformed by the spaces enriched by Timoshenko solutions, which are the only ones able to capture the oscillations of the rotation $\theta$.

In Figure 7 (a), the relative errors as the mesh is refined are plotted in terms of the resolution, incorporating the behavior of all the ninth-order spaces. The following observations are made: 

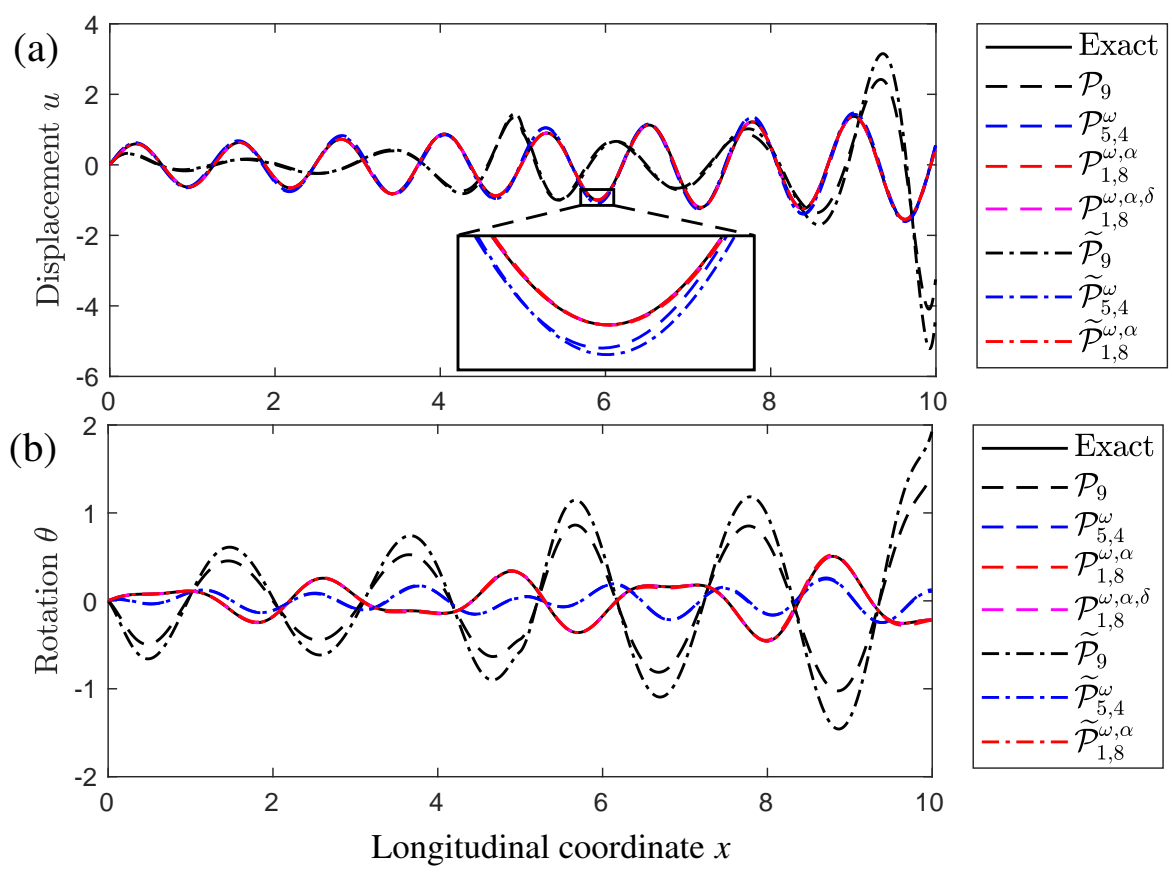

Figure 6: Exact and approached solutions for (a) the displacement $u$ and (b) the rotation $\theta$, for a cantilever beam with exponential profiles, for a circular frequency $\omega=5$ and $N_{\mathrm{e}}=2$ elements. The Timoshenko-enriched solutions (red and pink) are superposed with the exact one (solid black).

- For coarse meshes, the exponential-enriched space $\mathcal{P}_{1,8}^{\omega, \alpha, \delta}$ that embeds the analytical solution reaches the machine precision as expected (see below for refined meshes).

- The local rescaling does not affect the precision obtained with the polynomial space $\mathcal{P}_{9}$ and the sine-enriched space $\mathcal{P}_{5,4}^{\omega}$. On the other hand, it improves drastically, by several orders of magnitude, the precision obtained with the Timoshenko-enriched space $\mathcal{P}_{1,8}^{\omega, \alpha}$. This is expected in this particular case, because the amplitude factor $\mathrm{e}^{-\delta x}$ of the solution is exactly accounted for by the local rescaling.

- For all enriched spaces, there is a threshold above which the conditioning of the discrete system penalizes the solution and the error begins to grow (see the discussion below). However, this threshold appears for small relative errors, lower than $10^{-8}$ for all the spaces in this example, and very acceptable errors can be obtained by choosing a medium-sized mesh. For instance, fixing $h=\lambda$ leads to relative errors varying between $10^{-4}\left(\mathcal{P}_{9}\right.$ and $\left.\widetilde{\mathcal{P}}_{9}\right)$ to $10^{-10}\left(\widetilde{\mathcal{P}}_{1,8}^{\omega, \alpha}\right)$.

Finally, the influence of the frequency on the performances of the enriched spaces is investigated. To this end, the errors obtained with a fixed regular mesh with $N_{\mathrm{e}}=10$ elements, i.e. $h=1$, and increasing frequencies are displayed in Figure 7 (b). Errors obtained with polynomial spaces increase regularly with $\omega$ (in fact, at rate $\omega^{9}$ i.e. as $(h / \lambda)^{9}$ as expected for ninth-order polynomial spaces [27]), until they reach a plateau. On the other hand, errors obtained with enriched spaces (i) increase as $\omega \rightarrow 0$ (i.e. as the resolution increases) due again to bad conditioning, (ii) reach a minimum value for medium resolution and frequency and (iii) increase more slowly than with $\mathcal{P}_{9}$ and finally seem to reach a plateau at low error levels. Combined with the Timoshenko-enriched space $\mathcal{P}_{1,8}^{\omega, \alpha}$, the local rescaling has a remarkable frequency-dependent effect that leads to a large improvement for high frequencies.

Distributed loading. As a second validation test, the same exponentially varying cantilever beam is considered, this time free at the right extremity, i.e. $(N, M)(L)=(0,0)$, but submitted to a distributed loading. A linear density of forces with amplitude $q_{\mathrm{P}}$ corresponding to a particular solution $\left(u_{\mathrm{P}}, \theta_{\mathrm{P}}\right)$, with $u_{\mathrm{P}}(x)=(x / L)^{2}$ and linear $\theta_{\mathrm{P}}$, is designed, see Appendix B This way, the exact solution writes $(u, \theta)=\left(u_{\mathrm{P}}, \theta_{\mathrm{P}}\right)+\left(u_{\mathrm{H}}, \theta_{\mathrm{H}}\right)$, where the "homogeneous" part $\left(u_{\mathrm{H}}, \theta_{\mathrm{H}}\right)$ satisfies the system (14) and therefore is a combination of the free vibration solutions (17). This example is 

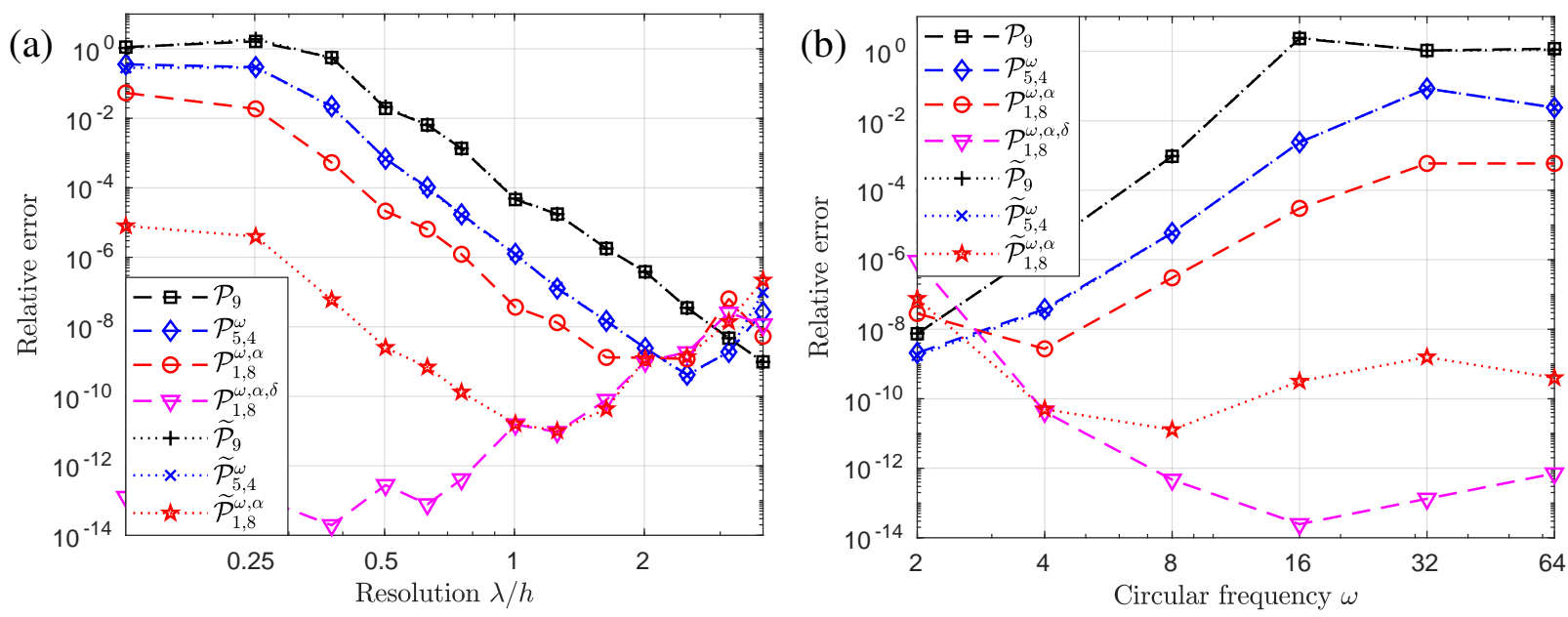

Figure 7: Relative errors for a cantilever beam with exponential profiles and $g=2.5$ : (a) fixed frequency $(\omega=5)$ and increasing resolution $\lambda / h$ $\left(N_{\mathrm{e}}=1\right.$ to 25 elements); and (b) fixed mesh $\left(N_{\mathrm{e}}=10\right.$ elements of length $\left.h=1\right)$ and increasing frequency.

chosen so that none of the FE spaces contains the full solution: $\mathcal{P}_{9}$ and $\mathcal{P}_{5,4}^{\omega}$ contain the particular solution but not the homogeneous part, and $\mathcal{P}_{1,8}^{\omega, \alpha, \delta}$ contains the homogeneous part but not the particular solution.

Remark 8. To address this specific loading, it would be easy to build a tenth-order space $\mathcal{P}_{2,8}^{\omega, \alpha, \delta}$ by adding an additional second-order polynomial shape function to the elementary bases of $\mathcal{P}_{1,8}^{\omega, \alpha, \delta}$, and therefore recover a space that contains the exact solution. More generally, when a particular solution is known exactly or approximately, higherorder FE spaces can be designed by adding relevant additional shape functions to the bases of existing spaces, using the half-hat $P U$.

This solution $(u, \theta)$ is plotted in Figure 8 for $\omega=5$, along with the approximations obtained with the studied spaces and for $N_{\mathrm{e}}=3$ elements. Again, for this configuration the enriched spaces clearly outperforms the polynomial space, especially for the approximation of the rotation $\theta$. Figure 9 presents the convergence of the errors for fixed frequency and then fixed mesh. Contrarily to the previous example, all the enriched spaces present similar performances, due to the additional force density. The local rescaling, which was designed by studying the Timoshenko system without source terms, still provides a slight error decrease.

Conditioning and static condensation. Finally, to better understand the conditioning effects, Figure 10 gives the conditioning number of the matrices $\boldsymbol{K}-\omega^{2} \boldsymbol{M}$ (for the original formulation) and $\widetilde{\boldsymbol{K}}-\widetilde{\boldsymbol{D}}-\omega^{2} \widetilde{\boldsymbol{M}}$ (for the modified formulation) for the frequency $\omega=5$ and the meshes used in the two previous examples (Figures 7 (a) and 9 a)). In Figure 10.a) are plotted the average conditioning numbers of the $8 \times 8$ inner elementary matrices that gather the contributions of the inner functions for each element. As the definition of the elementary polynomial basis does not depend on the element size, the conditioning of the resulting matrices is almost insensitive to the resolution. On the contrary, the inner functions $\varphi_{\mathrm{e}}^{m \pm}$ incorporated in the enriched bases become nearly linearly dependent as the resolution increases, and the conditioning number of the matrices explodes. This bad conditioning is reflected on the global matrices as seen on Figure 10(b) and penalizes the accuracy of the solution for thin meshes $(h<\lambda)$, as noticed above in Figures 7 and 9 .

This bad conditioning is partially addressed by applying the static condensation method presented in Section 5.1 . the condensed global matrices are not only smaller, but also well conditioned for all FE bases, as seen in Figure 10.c). However, in this case the bad conditioning of elementary matrices still affects the accuracy of the solution, and results nearly identical to those displayed in Figures 7 and 9 (not plotted for brevity) were obtained with the equivalent condensed system. Since the considered linear systems are sufficiently small-sized to be solved rapidly without condensation, the full uncondensed matrices are used in the upcoming examples for simplicity. 

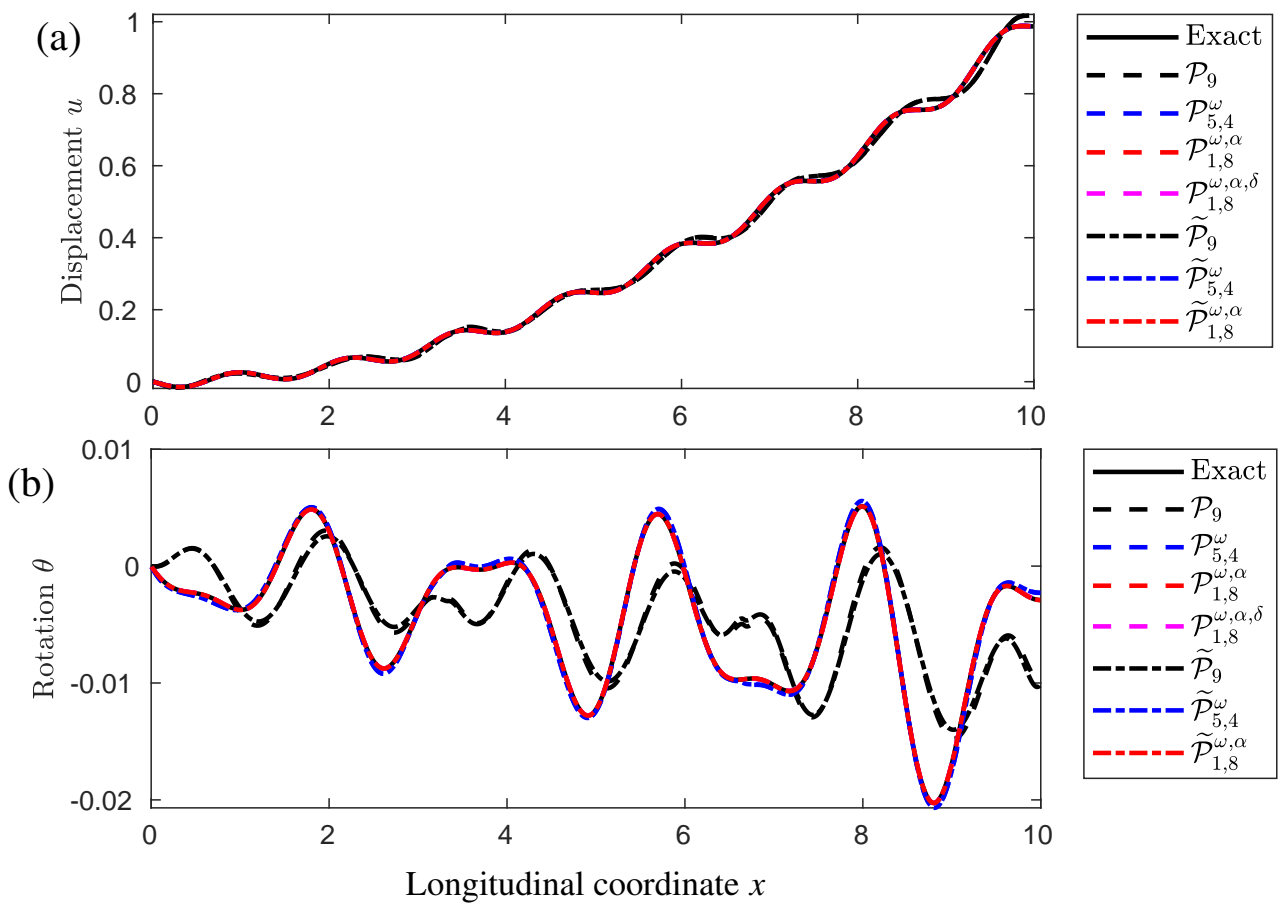

Figure 8: Exact and approached solutions for (a) the displacement $u$ and (b) the rotation $\theta$, for a cantilever beam with exponential profiles submitted to a distributed loading $q \neq 0$, for a circular frequency $\omega=5$ and $N_{\mathrm{e}}=3$ elements. The Timoshenko-enriched solutions (red and pink) are superposed with the exact one (solid black).
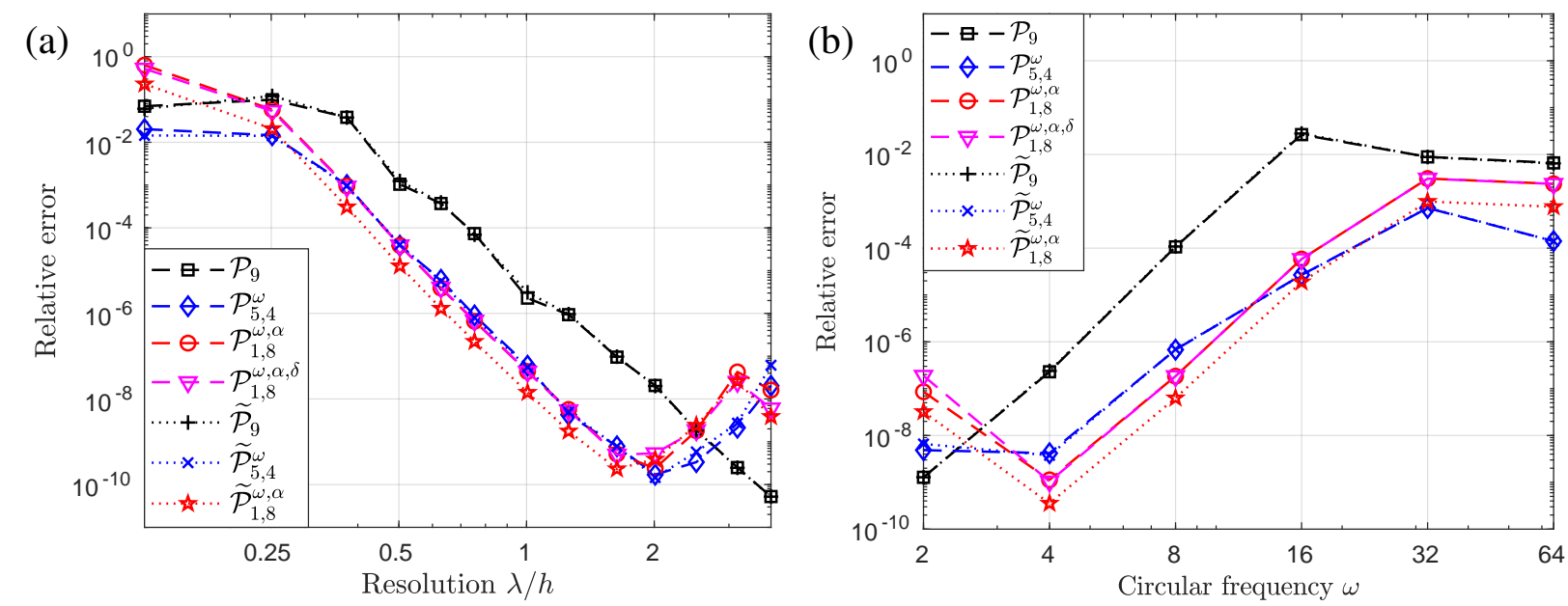

Figure 9: Relative errors for a cantilever beam with exponential profiles and $g=2.5$, submitted to a distributed loading $q \neq 0$ : (a) fixed frequency $\omega=5$ and increasing resolution $\lambda / h\left(N_{\mathrm{e}}=1\right.$ to 25 elements); and (b) fixed mesh $\left(N_{\mathrm{e}}=10\right.$ elements of length $\left.h=1\right)$ and increasing frequency.

\subsection{Comparison of the FE methods for four heterogeneous beams}

To show the efficiency of the method combining enriched elements and local rescaling to handle the inhomogeneity of the beam, results obtained for four cantilever beams are now presented. These beams feature several geometries for which no analytical solutions are available, ordered by increasing complexity of the inhomogeneity:

1. a beam with constant thickness and quadratically varying width (so that $\mathcal{A}=\mathcal{I}$ ),

2. a conical beam with circular cross-section and linearly decreasing radius (so that $\mathcal{A} \neq \mathcal{I}$ but the profiles are still smoothly and simultaneously decreasing), 

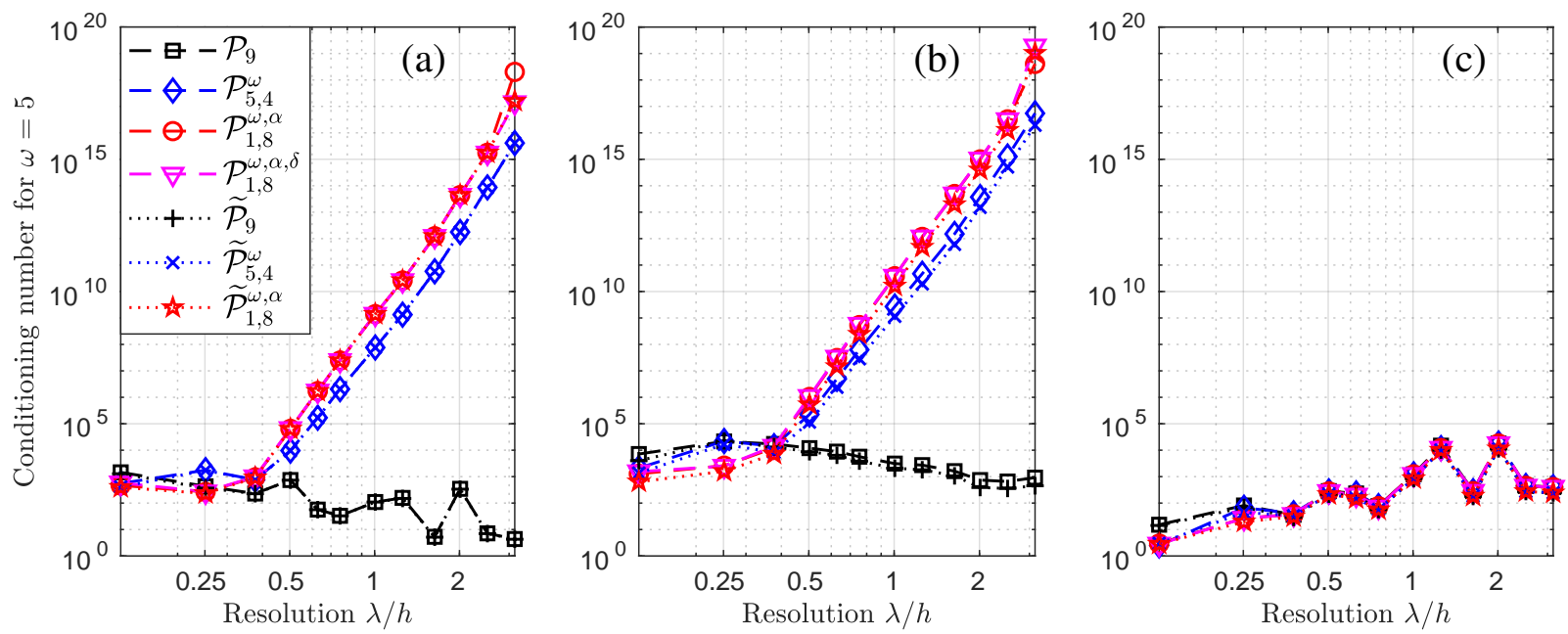

Figure 10: Conditioning numbers of the FE matrices corresponding to the results of Figure 7 a) obtained for an exponentially varying beam, for fixed frequency $\omega=5$ and decreasing meshsize: (a) elementary $8 \times 8$ matrices corresponding to inner shape functions (the conditioning number is averaged over all the elements); (b) matrices of the systems 24 , and 43 , without condensation; and (c) condensed matrices.

3. a beam with oscillating thickness and width (so that the profiles oscillate with different periods),

4. a periodically notched beam with non-smooth profiles (so that the mesh needs to be adapted to the singularities).

For each of these beams, only the "point-like" loading case is considered, i.e. the boundary conditions are set to $(u, \theta)(0)=(0,0)$ and $(N, M)(L)=(1,0)$. For each considered circular frequency $\omega$, a reference solution $\left(u_{\text {ref }}, \theta_{\text {ref }}\right)$ is computed using the polynomial space $\mathcal{P}_{9}$ and a very thin mesh made of $\lceil 4 \omega\rceil$ elements per unit length, i.e. elements of length $h=\lambda / 8 \pi$ for integer values of $\omega$. For instance, for the computations performed on beams of length $L=10$ at frequency $\omega=5$, the reference solution is computed with $N_{\mathrm{e}}=200$ elements. This resolution produced errors close to machine precision for tests performed with homogeneous and exponentially varying beams. Then, in the computations of the Lagrangian-based error $E_{\mathcal{L}}$ defined by (47), the exact solution $(u, \theta)$ is replaced by these reference solutions $\left(u_{\text {ref }}, \theta_{\text {ref }}\right)$.

\subsubsection{Beam with constant thickness and quadratically varying width}

The first example concerns a beam with rectangular cross-section, constant thickness and quadratically varying width. With the scaling $A_{\mathrm{c}}=A(0)$ and $I_{\mathrm{c}}=I(0)$, one has:

$$
\mathcal{A}(x)=\mathcal{I}(x)=(1+b x / L)^{2},
$$

with $b=\sqrt{\mathcal{A}(L)}-1$. Choosing $L=10$ and $\mathcal{A}(L)=1 / 8$, one obtains the beam and profiles represented in Figure 11 .

This beam is very similar to the exponentially varying beam, and the convergence results discussed above are retrieved in Figure 12, with the following differences. First, using the space $\mathcal{P}_{1,8}^{\omega, \alpha, \delta}$ instead of $\mathcal{P}_{1,8}^{\omega, \alpha}$, i.e. enrichment functions corresponding to exponentially varying beams instead of homogeneous beams, brings a stable improvement: the error is divided by a factor $\approx 2$. A similar improvement was already observed for bars in the previous work [39]. On the other hand, the local rescaling improves the precision obtained with $\mathcal{P}_{1,8}^{\omega, \alpha}$ by several orders of magnitude.

For the next examples, on the basis of this observation, the results obtained with the space $\mathcal{P}_{1,8}^{\omega, \alpha}$ are not displayed: the focus is instead on the space $\mathcal{P}_{1,8}^{\omega, \alpha}$ (which is locally enriched for variable width, see Section 3.4 ) and its counterpart $\widetilde{\mathcal{P}}_{1,8}^{\omega, \alpha}$ incorporating the rescaling.

\subsubsection{Conical beam}

The second example addresses a conical beam with circular cross-section, whose radius $\bar{r}(\bar{x})$ varies linearly: $\bar{r}(\bar{x})=\bar{r}_{0}+\left(\bar{r}_{L}-\bar{r}_{0}\right) \bar{x} / \bar{L}$ for some $\left(\bar{r}_{0}, \bar{r}_{L}, \bar{L}\right)$. Recalling that in this case $A=\pi \bar{r}^{2}$ and $I=\pi \bar{r}^{4} / 4$, using again the scaling $A_{\mathrm{c}}=A(0)$ and $I_{\mathrm{c}}=I(0)$ (so that $r_{\mathrm{c}}=\bar{r}_{0} / 2$ ), one obtains:

$$
\mathcal{A}(x)=(1+b x / L)^{2} \text { and } \mathcal{I}(x)=(1+b x / L)^{4},
$$


(a)

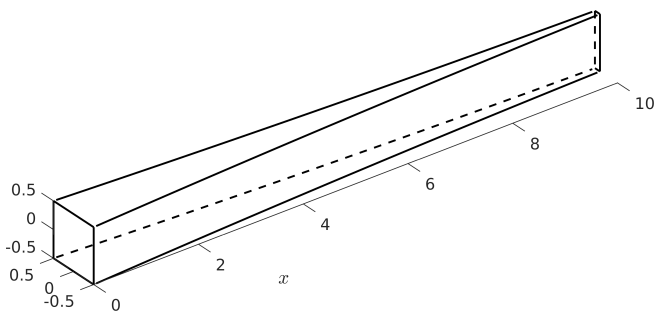

(b)

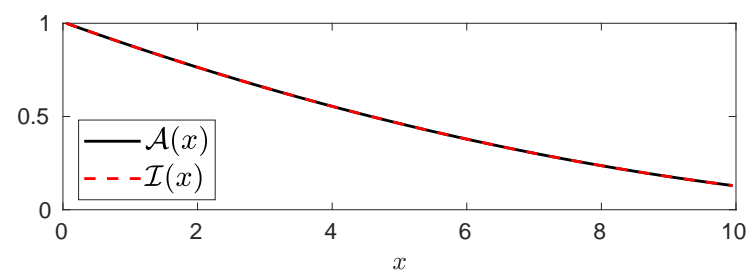

Figure 11: Beam with constant thickness and quadratic width: (a) representation and (b) profiles.
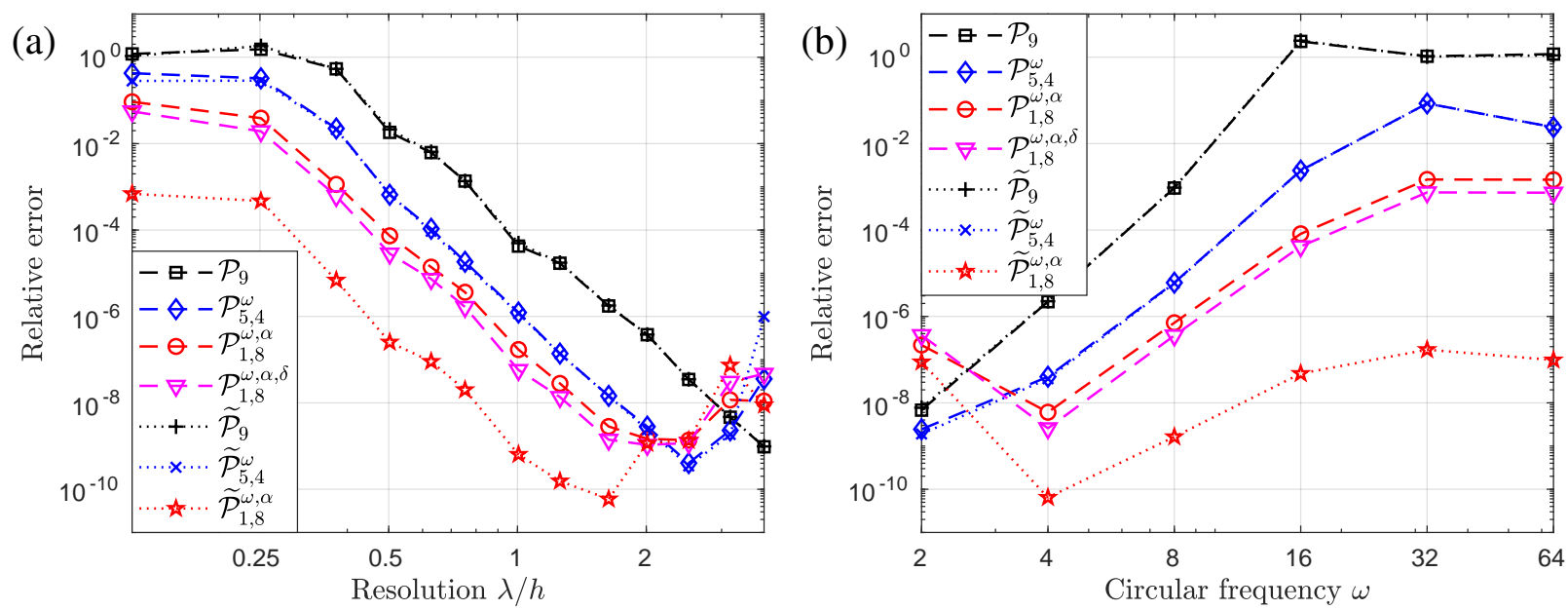

Figure 12: Relative errors for a cantilever beam with quadratic profiles represented in Figure 11 and $g=2.5$ : (a) fixed frequency $\omega=5$ and increasing resolution $\lambda / h\left(N_{\mathrm{e}}=1\right.$ to 25 elements); and (b) fixed mesh $\left(N_{\mathrm{e}}=10\right.$ elements of length $\left.h=1\right)$ and increasing frequency.

with $b=\left(\bar{r}_{L}-\bar{r}_{0}\right) / \bar{r}_{0}$. Choosing $L=10$ and $b$ such that $\mathcal{A}(L)=1 / 8$ as in the previous example, the resulting beam and profiles are represented in Figure 13. Similar "tapered" beams are studied in [13, 14].

Again, in Figure 14 is it seen that enriched spaces perform better than the polynomial space, but for this example the gap is thinner between the Timoshenko-enriched and the sine-enriched spaces. The change of unknowns still does not affect the performances of the polynomial and sine-enriched spaces, but enables to gain an additional stable factor on the error (between 2 and 3 ) while using Timoshenko-enriched spaces.

The gap between the various errors becomes wider as the frequency increases, as displayed in Figure 14 (b), and the same remarks than for the previous example can be done: the errors obtained with enriched spaces increase more slowly than with the polynomial space, and reach a plateau for error values smaller than $1 \%$ for the Timoshenkoenriched space. The gain brought by the modified formulation stays noticeable but not as significant as it was for the previous example.

(a)

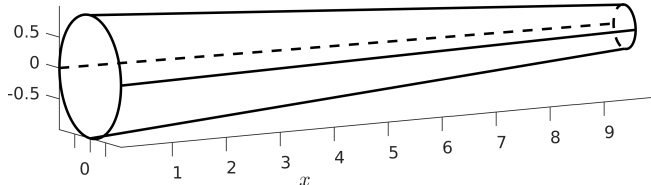

(b)

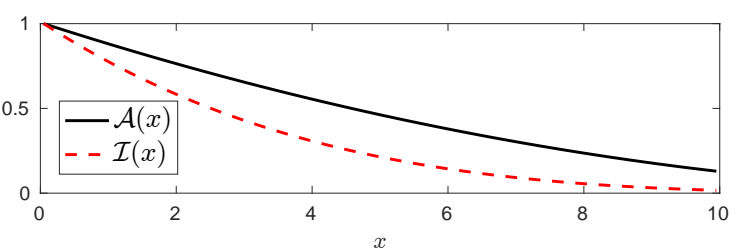

Figure 13: Conical beam with linearly varying radius: (a) representation and (b) profiles. 

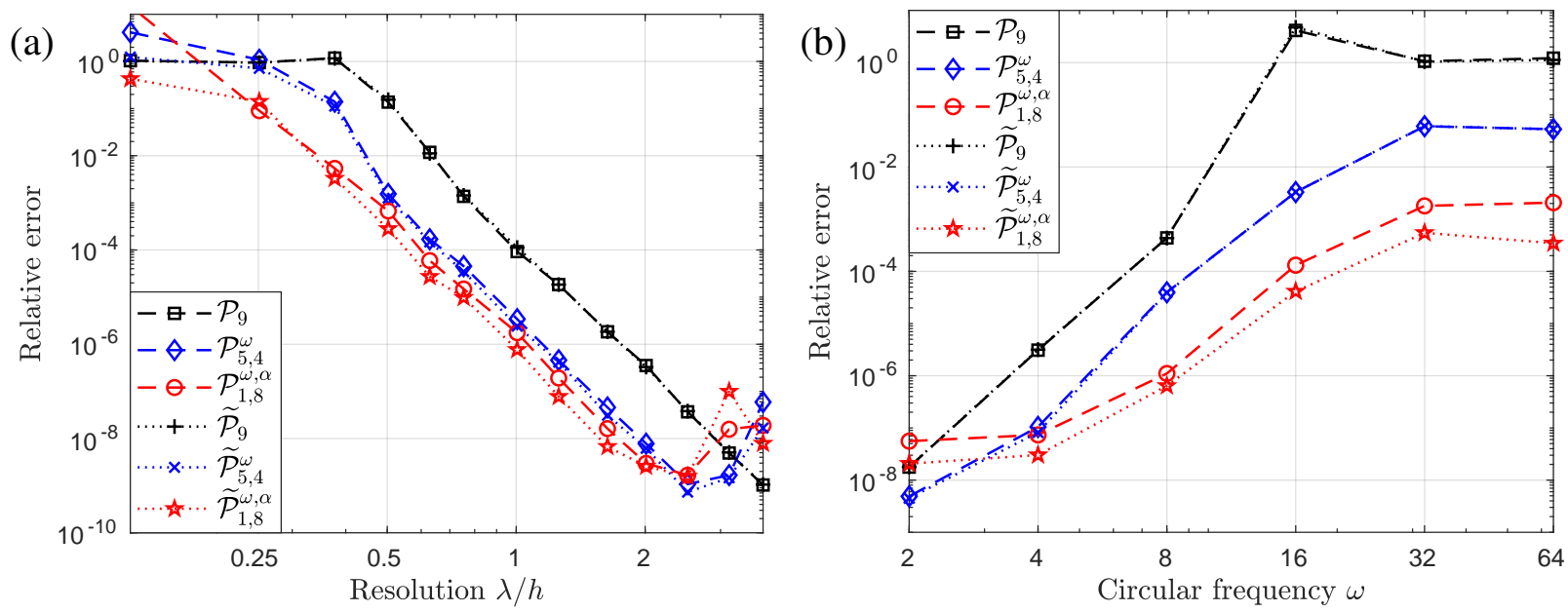

Figure 14: Relative errors for the conical cantilever beam represented in Figure 13 and $g=2.5$ : (a) fixed frequency $\omega=5$ and increasing resolution $\lambda / h\left(N_{\mathrm{e}}=1\right.$ to 25 elements $)$; and (b) fixed mesh $\left(N_{\mathrm{e}}=10\right.$ elements of length $\left.h=1\right)$ and increasing frequency.

\subsubsection{Beam with oscillating thickness and width}

The third example concerns again a beam with rectangular cross-sections, whose profiles $\mathcal{A}$ and $\mathcal{I}$ are defined in terms of the dimensionless width $b_{2}$ and thickness $b_{3}$ as:

$$
\mathcal{A}(x)=b_{2}(x) b_{3}(x) \quad \text { and } \quad \mathcal{I}(x)=b_{2}(x)\left(b_{3}(x)\right)^{3} .
$$

More specifically, oscillatory functions are chosen for these geometric parameters: $b_{2}(x)=1-0.5 \sin (2 \pi x / \ell)$ and $b_{3}(x)=1+0.5 \sin (2 \pi x / \ell)$. In this way, $\mathcal{A}$ remains close to 1 , while $\mathcal{I}$ oscillates with a wider amplitude as represented in Figure 15.(b) for $L=10$ and $\ell=6$.

As seen in Figure 16(a), for the fixed low-frequency simulation the improvement brought by the enriched spaces is lower than in the previous examples. On the other hand, for this example the local rescaling improves the results obtained with all spaces: in the convergence regime the error obtained using the polynomial space (resp. enriched spaces) is reduced by a factor $\approx 2$ (resp. $\approx 10)$.

As displayed in Figure 16(b), when the mesh is fixed and the frequency increases, this overall improvement progressively disappears and the Timoshenko-enriched space remains the only one to benefit from its association with the modified formulation, as observed on the previous examples.

(a)

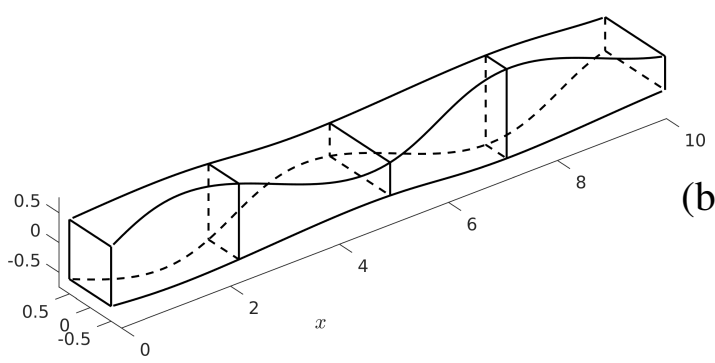

(b)

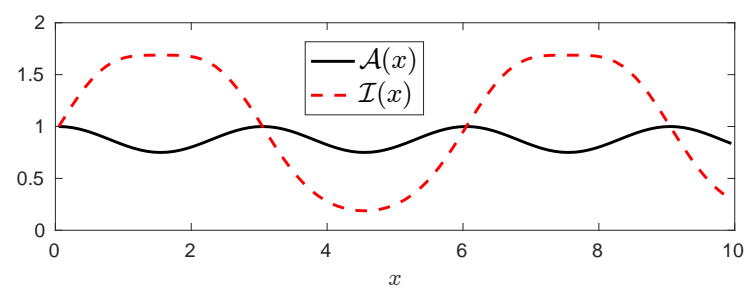

Figure 15: Beam with rectangular cross-sections and oscillating thickness and width: (a) representation and (b) profiles.

\subsubsection{Periodically notched beam}

The last example, inspired by the work [9], concerns a periodically notched beam represented in Figure 18(a), made of six straight segments and five notches with constant width and varying thickness as specified in Figure 17 . In this case, before the mesh definition, the beam is divided into subdomains whose boundaries match the singularities 

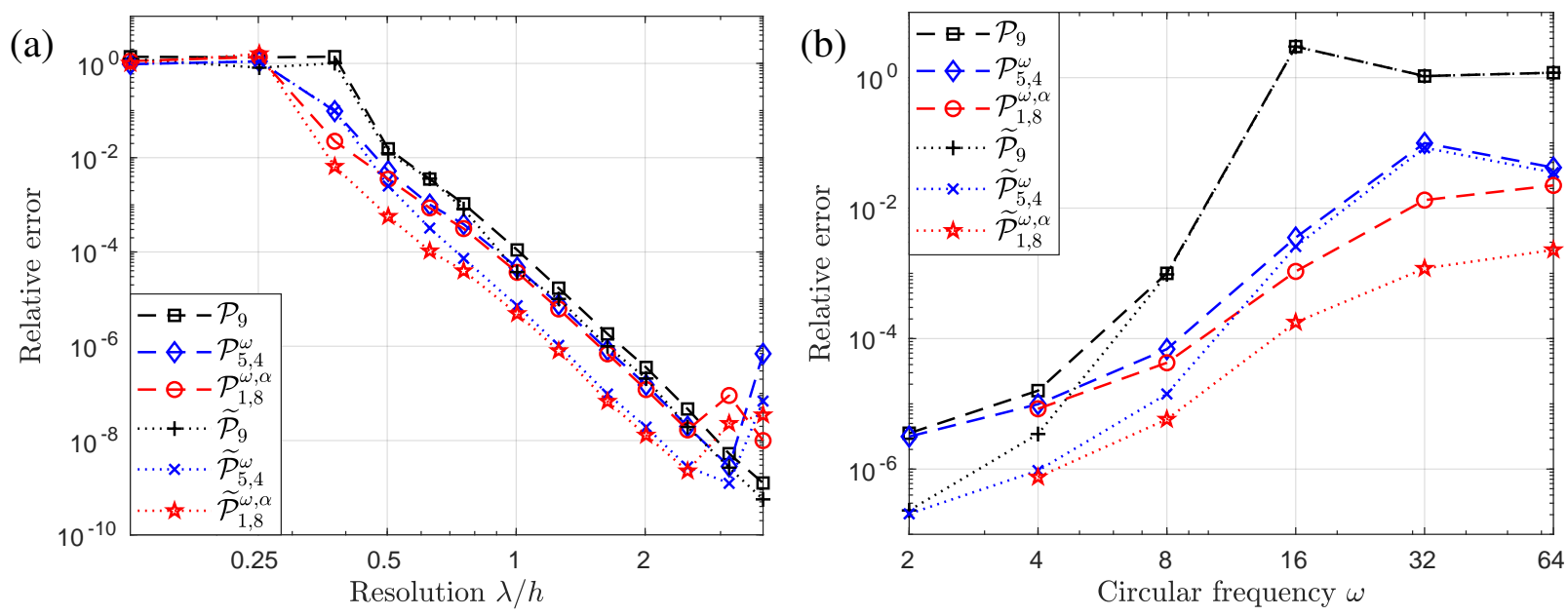

Figure 16: Relative errors for the cantilever beam with oscillating profiles represented in Figure 15 and $g=2.5$ : (a) fixed frequency $\omega=5$ and increasing resolution $\lambda / h\left(N_{\mathrm{e}}=1\right.$ to 25 elements); and (b) fixed mesh $\left(N_{\mathrm{e}}=10\right.$ elements of length $\left.h=1\right)$ and increasing frequency.

of the profiles, i.e. there is one subdomain per straight segment and two per notch. Each subdomain is meshed independently.

The figure 19 a) illustrates the convergence rate obtained as the resolution increases for a fixed frequency $\omega=50$ and regular meshes. A high frequency was chosen to avoid the high-resolution and badly-conditioned configurations, since regular meshes must have at least 34 elements. One observes again the stable improvements brought by the enrichment strategy and the rescaling.

For the convergence study with fixed mesh, the coarsest possible mesh is chosen to demonstrate the capabilities of the method: each subdomain contains only one element, for a total of only $N_{\mathrm{e}}=16$ elements (corresponding to the subdivision displayed in Figure 18 (a)). This coarse mesh is particularly justified for the straight subdomains since the exact local solution is then embedded in the Timoshenko-enriched basis of the space $\mathcal{P}_{1,8}^{\omega, \alpha}$. As seen in Figure 19. b), the polynomial and sine-enriched spaces are largely outperformed by the Timoshenko-enriched spaces for high frequencies. Moreover, combining the modified formulation with Timoshenko-enriched bases results in a very acceptable relative error (less than $0.05 \%$ ) on the whole interval of frequencies $\omega \in[2,64]$.

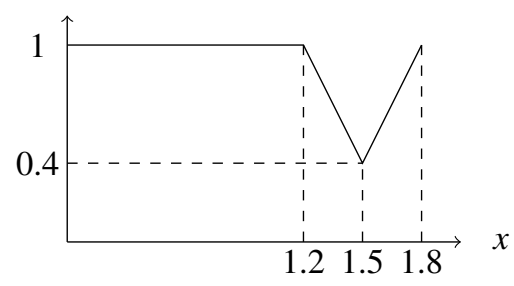

Figure 17: Variations of the thickness throughout the unit cell of the periodically notched beam.

\section{Discussion}

The numerical experiments of the previous section demonstrated the usefullness of the proposed enriched FEM to tackle time-harmonic Timoshenko problems, for various inhomogeneous beams. To complete these findings, possible extensions are now discussed, notably (i) theoretical investigations that could assess the properties of the method in a more general setting and (ii) applications to eigenfrequency search and time-domain simulations.

Theoretical investigations. An analysis of the method should lead to a better understanding of the respective contributions of its two components. The analysis of the enrichment with oscillatory functions would benefit on the many 
(a)

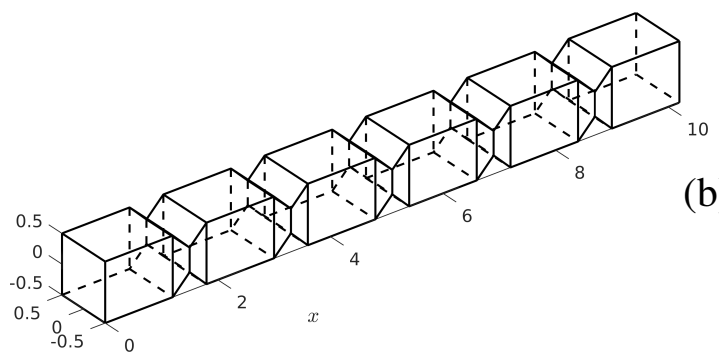

(b)

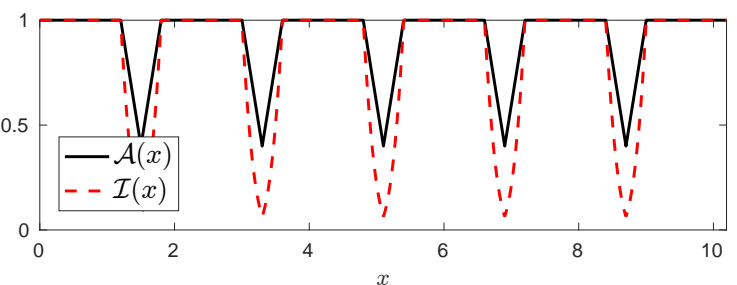

Figure 18: Periodically notched beam: (a) representation and (b) profiles.
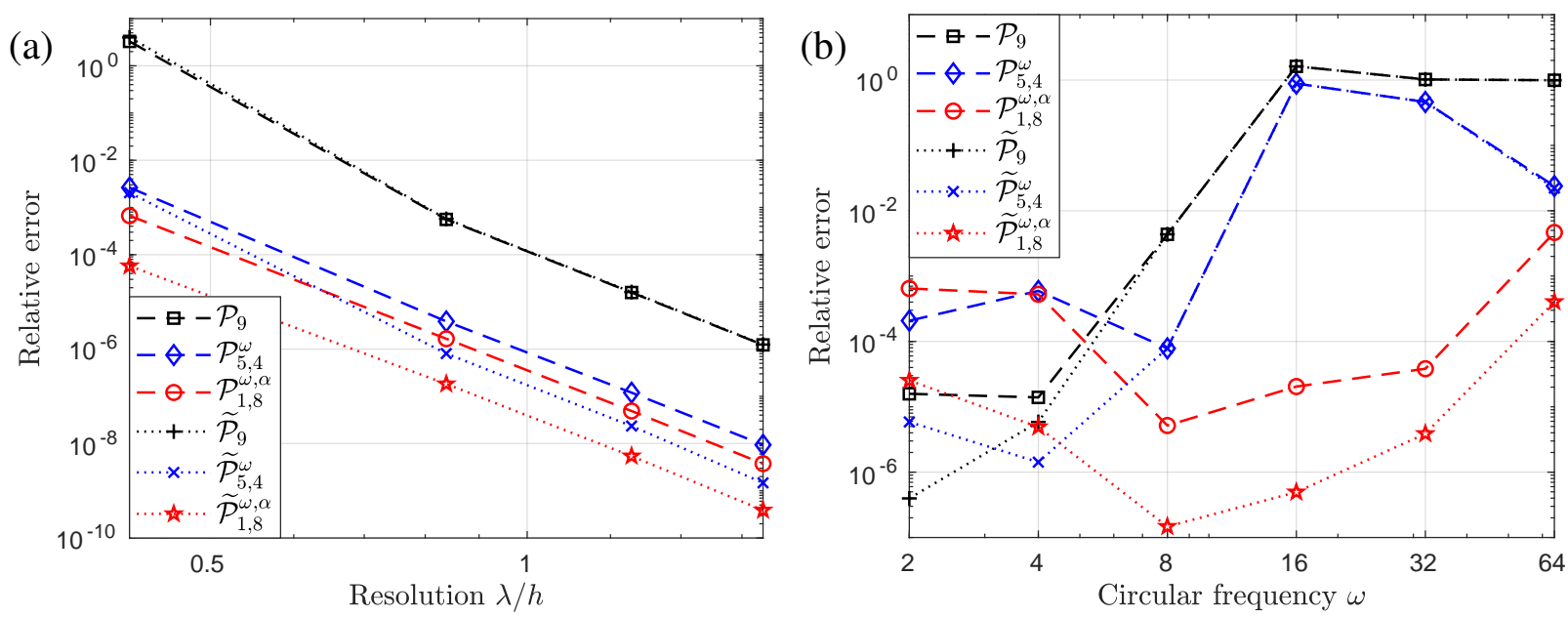

Figure 19: Relative errors for the periodically notched beam represented in Figure 18, with $g=2.5$ : (a) fixed frequency $(\omega=50)$ and increasing resolution $\lambda / h$ (regular meshes composed of $4 n$ elements per straight subdomain and $2 n$ elements per notch, i.e. $N_{\mathrm{e}}=34 n$ and $h=0.3 / n$, with $n=1 \ldots 4$.); and (b) fixed mesh (coarsest irregular mesh: one element of length $h_{1}=1.2$ per straight subdomain and two elements of length $h_{2}=0.3$ per notch, for a total of $N_{\mathrm{e}}=16$ elements) and increasing frequency.

results on plane wave enrichment for Helmholtz-like problems, see [39] and the references therein. Accounting for the several wavenumbers associated with the Timoshenko system at a fixed frequency would be an additional difficulty. One may also seek other relevant enrichment functions, e.g. generalized plane waves [44], and complete first attempts in this direction presented in Appendix A The analysis of the local rescaling should enable to propose $a$ priori estimates of the error reduction for given profile functions of a beam.

Computing natural frequencies of beams. Many papers on Timoshenko beams focus on the computations of their natural frequencies. Numerous methods are proposed [3] to build stiffness and mass matrices and obtain approximations $\omega_{h}$ of these frequencies as the solutions of the generalized eigenvalue problem:

$$
\text { Find }\left(\omega_{h}, \boldsymbol{U}\right) \text { such that }\left(\boldsymbol{K}-\omega_{h}^{2} \boldsymbol{M}\right) \cdot \boldsymbol{U}=\mathbf{0} .
$$

The enriched bases presented in this work are adapted to solve vibration problems at a specific frequency $\omega$, and therefore a priori seem poorly adapted to the search of natural frequencies. However, a procedure similar to the one developed for bars by Arndt et al. [23] could be investigated. Such procedure aims at determining precisely natural frequencies by (i) running an eigenfrequency search with a traditional piecewise-polynomial FE basis, and (ii) enrich the FE basis with functions corresponding to a frequency determined at step (i), to include functions close to the mode shape in the approximation space and improve the accuracy of the eigenvalue approximation for this particular frequency. Finally, the step (ii) can be repeated with the newly computed eigenfrequencies to improve the precision. 
Time-domain wave propagation in Timoshenko beams. Time-domain computation can be achieved in two ways. On the one hand, a numerical scheme can be used to discretize the considered time interval. In this case, the mass matrix should be inverted at every time step. The spectral finite element method, which produces diagonal mass matrices, is particularly adapted in this case [30, 28]. In contrast, the enriched FEM produces non-diagonal mass matrices; moreover it is built to be efficient for one particular frequency, thus it is not expected to be very efficient to retrieve solutions of a time-domain problem with a wide frequency spectrum.

On the other hand, one can apply a discrete Laplace transform [10, 15] or a Fourier transform [2, 25] to the timedomain problem, then solve the resulting time-harmonic problems in the Laplace or Fourier domain for a relevant range of frequencies, and finally come back to the time-domain by an inverse transform. For this approach, the proposed enriched bases may be useful: at each frequency the computation could be accelerated by coarsening the mesh and using an appropriate enrichment, at the cost of computing the $\omega$-dependent stiffness and mass matrices. In view of the results obtained in Section 5 for fixed meshes and increasing frequencies, one may even use the same mesh for a wide range of frequencies while keeping reasonable error levels, which could facilitate parallel computations and post-processing operations.

\section{Conclusions}

In this paper, a finite element approach dedicated to the computation of vibrations of heterogeneous beams is presented. The method relies on (i) an enrichment method incorporating solutions of Timoshenko systems into the approximation space and (ii) a local rescaling that accounts for the heterogeneous geometry. The resulting discrete solutions were compared with solutions obtained using classical polynomial FE spaces on several examples. With the same implementation easiness and similar computational costs, the proposed enriched FEM outperforms the polynomial FEM on the tested configurations, especially when coupled with the local rescaling. On the one hand, for a fixed frequency, all errors converge with the same rate as the mesh is refined, but using the enriched FEM improves the precision by a factor ranging from about one to several orders of magnitude depending on the considered geometry. On the other hand, using the enriched FEM enables to use the same coarse mesh to solve time-harmonic problems in a wide range of frequencies (several octaves), while keeping the error level at very acceptable levels (typically less than $0.1 \%$ ), whereas the polynomial FEM fails to follow the fast oscillations of the solutions at high frequencies without mesh refinement. Several extensions and applications of the method were finally discussed.

\section{Acknowledgments}

The authors acknowledge the support of the Centre Henri Lebesgue ANR-11-LABX-0020-01 provided through a post-doctoral fellowship to R. Cornaggia.

\section{Appendix A. Other enriched spaces}

This appendix presents other attempts made to define relevant enrichment families, that produced no noticeable improvement compared to the spaces presented in Sections 3.4 and 4.3 , for the examples of Section 5 .

Enrichment family resulting from the Taylor expansion of Timoshenko system for arbitrary profiles. To build relevant enrichment functions for a given equation with varying coefficients, an idea proposed by Imbert-Gérard and Després [44] (who study Helmholtz equations with variable wavenumbers) is to replace these coefficients by their Taylor expansions about the middle $x_{e}$ of an element and look for the solutions of the obtained equations, called generalized plane waves [44]. Here, denoting $(\alpha, 2 \delta, 2 \beta)$ the values of the coefficients of the system [5] at the middle-point $x_{e}$ (i.e. their 0-th order Taylor expansion):

$$
\alpha=\frac{\mathcal{A}}{\mathcal{I}}\left(x_{e}\right), \quad 2 \delta=\frac{\mathcal{A}^{\prime}}{\mathcal{A}}\left(x_{e}\right), \quad \text { and } \quad 2 \beta=\frac{\mathcal{I}^{\prime}}{\mathcal{I}}\left(x_{e}\right),
$$

and keeping only these leading-order contributions in the equations $[5]$, the resulting system is:

$$
\left\{\begin{array}{l}
-\omega^{2} u=2 \delta\left(u^{\prime}-\theta\right)+\left(u^{\prime}-\theta\right)^{\prime} \\
-\omega^{2} \theta=\alpha\left(u^{\prime}-\theta\right)+g\left(2 \beta \theta^{\prime}+\theta^{\prime \prime}\right) .
\end{array}\right.
$$


This system does not correspond to an actual beam when $\delta \neq \beta$ and therefore its relevance is hard to determine using physical insights. The dispersion relation is found to be:

$$
\left(\omega^{2}-\left(k^{2}-2 \mathrm{i} \delta k\right)\right)\left(\omega^{2}-g\left(k^{2}-2 \mathrm{i} \beta k\right)-\alpha\right)-\alpha\left(k^{2}-2 \mathrm{i} \delta k\right)=0,
$$

or:

$$
g k^{4}-2 g \mathrm{i}(\delta+\beta) k^{3}-\left(\omega^{2}(1+g)+4 g \delta \beta\right) k^{2}+2 \mathrm{i} \omega^{2}(\delta+g \beta) k+\omega^{2}\left(\omega^{2}-\alpha\right)=0 .
$$

The roots $\left\{\tilde{k}_{m}\right\}_{m=1 \ldots 4}$ of this equation may be computed numerically for each set of parameters $(\omega, \alpha, \delta, \beta)$, and the basis of solutions is then given by:

$$
\Psi^{\omega, \alpha, \delta, \beta}=\left\{x \mapsto \mathrm{e}^{\mathrm{i} \tilde{k}_{m} x}\right\}_{m=1 \ldots 4} .
$$

This family was used to build a space $\mathcal{P}_{1,8}^{\omega, \alpha, \delta, \beta}$ similar to $\mathcal{P}_{1,8}^{\omega, \alpha}$ and $\mathcal{P}_{1,8}^{\omega, \alpha, \delta}$, for which the $e$-th elementary basis is determined from the values of $(\alpha, \delta, \beta)$ computed with $(\mathrm{A} .1)$. However, no significant improvement was observed compared to the simpler space $\mathcal{P}_{1,8}^{\omega, \alpha}$, and compared to $\mathcal{P}_{1,8}^{\omega, \alpha, \delta}$ for beams with constant thickness.

The same idea was applied to the system (41) arising after the change of unknowns $(u, \theta) \rightarrow(\tilde{u}, \tilde{\theta})$. By retaining only the middle-point values of all the variable coefficients $\left(d^{\prime \prime} / d, d^{\prime} / a, \ldots\right)$, writing the dispersion relation of the resulting system and computing its roots, one may define a new family $\Psi^{\omega, d, a}$ of enrichment functions and build the corresponding space $\widetilde{\mathcal{P}}_{1,8}^{\omega, d, a}$ similarly than above. This space was used instead of $\widetilde{\mathcal{P}}_{1,8}^{\omega, \alpha}$ to discretize the modified formulation (42), but again, no clear improvement was observed.

Enrichments inspired by the Stable generalized FEM. Given an enrichment family $\left\{\psi_{m}\right\}$, there are alternative ways to add inner functions (i.e. functions that vanish at both nodes of the element) to an elementary basis. In particular, one of the ideas of the Stable GFEM [33] is to substract the linear interpolant of the enrichment functions on each element. Using this idea, alternative Timoshenko-enriched spaces $\mathcal{P}_{5,4}^{\omega, \alpha}$ were built, for which only four additional inner functions are added to a fifth-order polynomial basis. These functions read:

$$
\overline{\varphi_{\mathrm{e}}^{m}}(\xi)=\mathrm{e}^{\mathrm{i} k_{m} h_{e} \xi}-1-\xi\left(\mathrm{e}^{\mathrm{i} k_{m} h_{e}}-1\right), \quad m=1 \ldots 4,
$$

where the wavenumbers $k_{m}$ are defined by (12). In particular, one may expect these bases to produce better-conditioned systems (as each enrichment function appears only once in the elementary basis). However, on the examples presented in Section 5 these spaces were found to perform poorly compared to the spaces $\mathcal{P}_{1,8}^{\omega, \alpha}$, that uses the same enrichment functions but multiplied by the "half-hat" local PU.

\section{Appendix B. An exact solution for nonzero density of forces}

For an exponential beam, a particular solution of the Timoshenko system $(5)$ is sought by imposing $u_{\mathrm{P}}(x)=(x / L)^{2}$ and $m(x)=0$. Then the unknown rotation $\theta_{\mathrm{P}}$ and density of forces $q_{\mathrm{P}}(x)=\mathcal{A}(x) \widetilde{q}_{\mathrm{P}}(x)$ are the solutions of the system:

$$
\left\{\begin{array}{l}
u_{\mathrm{P}}^{\prime \prime}-\theta_{\mathrm{P}}^{\prime}+2 \delta\left(u_{\mathrm{P}}^{\prime}-\theta_{\mathrm{P}}\right)+\omega^{2} u_{\mathrm{P}}+\widetilde{q}_{\mathrm{P}}=0 \\
g\left(\theta_{\mathrm{P}}^{\prime \prime}+2 \delta \theta_{\mathrm{P}}^{\prime}\right)+\alpha\left(u_{\mathrm{P}}^{\prime}-\theta_{\mathrm{P}}\right)+\omega^{2} \theta_{\mathrm{P}}=0 .
\end{array}\right.
$$

The rotation $\theta_{\mathrm{P}}$ and the force density factor $\widetilde{q_{\mathrm{P}}}$ are determined successively thanks to the second and the first equation. Finally, the particular solution

$$
u_{\mathrm{P}}(x)=\frac{x^{2}}{L^{2}}, \quad \theta_{\mathrm{P}}(x)=\frac{1}{L^{2}} \frac{2 \alpha}{\alpha-\omega^{2}}\left(x+\frac{2 g \delta}{\alpha-\omega^{2}}\right)
$$

is obtained by imposing the linear density of forces:

$$
q_{\mathrm{P}}(x)=\mathcal{A}(x) \widetilde{q_{\mathrm{P}}}(x)=\frac{\mathrm{e}^{2 \delta x}}{L^{2}}\left[-\omega^{2} x^{2}+\frac{2}{\alpha-\omega^{2}}\left(2 \delta \omega^{2} x+\omega^{2}+\frac{4 g \alpha \delta^{2}}{\alpha-\omega^{2}}\right)\right] .
$$




\section{References}

[1] K. F. Graff, Wave motion in elastic solids, Oxford University Press, 1975.

[2] S. Gopalakrishnan, M. Martin, J. Doyle, A matrix methodology for spectral analysis of wave propagation in multiple connected Timoshenko beams, J. Sound Vib. 158 (1) (1992) 11 - 24. doi:10.1016/0022-460X (92)90660-P

[3] M. Hajianmaleki, M. S. Qatu, Vibrations of straight and curved composite beams: A review, Compos. Struct. 100 (2013) 218 - 232. doi: 10.1016/j.compstruct.2013.01.001

[4] W. Cleghorn, B. Tabarrok, Finite element formulation of a tapered Timoshenko beam for free lateral vibration analysis, J. Sound Vib. 152 (3) (1992) 461 - 470. doi:10.1016/0022-460X(92)90481-C

[5] X. Tong, B. Tabarrok, K. Yeh, Vibration analysis of Timoshenko beams with non-homogeneity and varying cross-section, J. Sound Vib. 186 (5) (1995) 821 - 835. doi:10.1006/jsvi.1995.0490

[6] D. Zhou, Y. K. Cheung, Vibrations of tapered Timoshenko beams in terms of static Timoshenko beam functions., ASME. J. Appl. Mech doi: 10.1115/1.1357164

[7] J. Katsikadelis, G. Tsiatas, Regulating the vibratory motion of beams using shape optimization, J. Sound Vib. 292 (1-2) (2006) $390-401$. doi:10.1016/j.jsv.2005.08.002

[8] N. Olhoff, B. Niu, G. Cheng, Optimum design of band-gap beam structures, Int. J. Solids Struct. 49 (22) (2012) 3158-3169. doi : 10.1016/ j.ijsolstr.2012.06.014

[9] A. Zak, M. Krawczuk, M. Palacz, L. Doliński, W. Waszkowiak, High frequency dynamics of an isotropic Timoshenko periodic beam by the use of the time-domain spectral finite element method, J. Sound Vib. 409 (2017) 318 - 335. doi:10.1016/j.jsv.2017.07.055

[10] V. Adàmek, F. Valeš, Analytical solution for a heterogeneous Timoshenko beam subjected to an arbitrary dynamic transverse load, Eur. J. Mech. A. Solids 49 (2015) 373 - 381. doi:10.1016/j.euromechsol.2014.07.016

[11] K. Sarkar, R. Ganguli, Closed-form solutions for axially functionally graded Timoshenko beams having uniform cross-section and fixed-fixed boundary condition, Compos. Part B: Eng. 58 (2014) 361 - 370. doi:10.1016/j.compositesb.2013.10.077

[12] Z. Wang, X. Wang, G. Xu, S. Cheng, T. Zeng, Free vibration of two-directional functionally graded beams, Compos. Struct. 135 (2016) 191 - 198. doi:10.1016/j.compstruct.2015.09.013

[13] A. Shahba, R. Attarnejad, M. T. Marvi, S. Hajilar, Free vibration and stability analysis of axially functionally graded tapered Timoshenko beams with classical and non-classical boundary conditions, Compos. Part B: Eng. 42 (4) (2011) 801 - 808. doi:10.1016/j. compositesb.2011.01.017

[14] Y. Huang, L.-E. Yang, Q.-Z. Luo, Free vibration of axially functionally graded Timoshenko beams with non-uniform cross-section, Compos. Part B: Eng. 45 (1) (2013) 1493 - 1498. doi:10.1016/j.compositesb.2012.09.015

[15] F. F. Calim, Transient analysis of axially functionally graded Timoshenko beams with variable cross-section, Compos. Part B: Eng. 98 (2016) 472 - 483. doi:10.1016/j.compositesb.2016.05.040

[16] M. Eisenberger, Dynamic stiffness matrix for variable cross-section Timoshenko beams, Commun. Numer. Methods Eng. 11 (6) (1995) 507-513. doi:10.1002/cnm.1640110605

[17] A.-Y. Tang, J.-X. Wu, X.-F. Li, K. Lee, Exact frequency equations of free vibration of exponentially non-uniform functionally graded Timoshenko beams, Int. J. Mech. Sci. 89 (2014) 1 - 11. doi:10.1016/j.ijmecsci.2014.08.017

[18] J. Yuan, Y.-H. Pao, W. Chen, Exact solutions for free vibrations of axially inhomogeneous Timoshenko beams with variable cross section, Acta Mech. 227 (9) (2016) 2625-2643. doi:10.1007/s00707-016-1658-6

[19] T. G. Ritto, R. Sampaio, E. Cataldo, Timoshenko beam with uncertainty on the boundary conditions, J. Braz. Soc. Mech. Sci. 30 (4) (2008) 295-303. doi:10.1590/s1678-58782008000400005

[20] D. Wu, W. Gao, K. Gao, F. Tin-Loi, Robust safety assessment of functionally graded structures with interval uncertainties, Compos. Struct. 180 (2017) 664-685. doi:10.1016/j.compstruct.2017.08.011

[21] G. C. Tsiatas, A. E. Charalampakis, Optimizing the natural frequencies of axially functionally graded beams and arches, Compos. Struct. 160 (2017) 256-266. doi:10.1016/j.compstruct.2016.10.057

[22] L. Tang, L. Cheng, Enhanced acoustic black hole effect in beams with a modified thickness profile and extended platform, J. Sound Vib. 391 (2017) 116-126. doi:10.1016/j.jsv.2016.11.010

[23] M. Arndt, R. Machado, A. Scremin, An adaptive generalized finite element method applied to free vibration analysis of straight bars and trusses, J. Sound Vib. 329 (6) (2010) 659 -672. doi:10.1016/j.jsv.2009.09.036

[24] M. Ostoja-Starzewski, A. Woods, Spectral finite elements for vibrating rods and beams with random field properties, J. Sound Vib. 268 (4) (2003) 779 - 797. doi:10.1016/S0022-460X(03)00037-3

[25] W. Shen, D. Li, S. Zhang, J. Ou, Analysis of wave motion in one-dimensional structures through fast-Fourier-transform-based wavelet finite element method, J. Sound Vib. 400 (2017) 369 - 386. doi:10.1016/j.jsv.2017.03.014

[26] A. Ern, J.-L. Guermond, Theory and practice of finite elements, Vol. 159, Springer Science \& Business Media, 2004.

[27] F. Ihlenburg, Finite Element Analysis of Acoustic Scattering, Springer Publishing Company, Incorporated, 1998.

[28] M. A. Sprague, T. L. Geers, Legendre spectral finite elements for structural dynamics analysis, Commun. Numer. Methods Eng. 24 (12) (2008) 1953-1965. doi:10.1002/cnm.1086

[29] C.-Y. Tai, Y. Chan, A hierarchic high-order Timoshenko beam finite element, Comput. Struct. 165 (2016) 48-58. doi:10.1016/j. compstruc.2015.12.002

[30] P. Kudela, M. Krawczuk, W. Ostachowicz, Wave propagation modelling in 1d structures using spectral finite elements, J. Sound Vib. 300 (1-2) (2007) 88 - 100. doi:10.1016/j.jsv.2006.07.031

[31] J. Melenk, I. Babuška, The partition of unity finite element method: Basic theory and applications, Comput. Methods Appl. Mech. Eng. 139 (1) (1996) 289 - 314. doi:10.1016/S0045-7825(96)01087-0

[32] T. Strouboulis, I. Babuška, K. Copps, The design and analysis of the generalized finite element method, Comput. Methods Appl. Mech. Eng. 181 (1-3) (2000) 43 - 69. doi:10.1016/S0045-7825(99) 00072-9 
[33] I. Babuška, U. Banerjee, Stable generalized finite element method (SGFEM), Comput. Methods Appl. Mech. Eng. 201-204 (2012) 91-111. doi:10.1016/j.cma.2011.09.012

[34] L. Li, Discretization of the Timoshenko beam problem by the p and the h-p versions of the finite element method, Numer. Math. 57 (1) (1990) 413-420. doi:10.1007/BF01386420

[35] I. Babuška, Z. Zhang, The partition of unity method for the elastically supported beam, Comput. Methods Appl. Mech. Eng. 152 (1) (1998) 1 - 18. doi: 10.1016/S0045-7825(97)00231-4

[36] J. Reddy, On locking-free shear deformable beam finite elements, Comput. Methods Appl. Mech. Eng. 149 (1) (1997) 113 - 132, containing papers presented at the Symposium on Advances in Computational Mechanics. doi : 10.1016/S0045-7825(97)00075-3

[37] Y. Shang Hsu, Enriched finite element methods for Timoshenko beam free vibration analysis, Appl. Math. Modell. 40 (15) (2016) 7012 7033. doi:10.1016/j.apm.2016.02.042

[38] T. Zhou, J.-D. Chazot, E. Perrey-Debain, L. Cheng, Performance of the partition of unity finite element method for the modeling of Timoshenko beams, Comput. Struct. 222 (2019) 148-154. doi:10.1016/j.compstruc.2019.07.004

[39] R. Cornaggia, E. Darrigrand, L. Le Marrec, F. Mahé, Enriched finite elements for time-harmonic Webster's equation, Comput. Methods Appl. Mech. Eng. 341 (2018) 985 - 1007. doi:10.1016/j.cma.2018.07.031

[40] F. Gruttmann, W. Wagner, Shear correction factors in Timoshenko's beam theory for arbitrary shaped cross-sections, Comput. Mech. 27 (3) (2001) 199-207. doi:10.1007/s004660100239

[41] C. Forgit, B. Lemoine, L. Le Marrec, L. Rakotomanana, A Timoshenko-like model for the study of three-dimensional vibrations of an elastic ring of general cross-section, Acta Mech. 227 (9) (2016) 2543-2575. doi :10.1007/s00707-016-1618-1

[42] F. Sohani, H. R. Eipakchi, Analytical solution for modal analysis of Euler-bernoulli and Timoshenko beam with an arbitrary varying crosssection, Math. Models Eng. 4 (3) (2018) 164-174. doi:10.21595/mme.2018.20116

[43] S. W. Rienstra, Webster's horn equation revisited, SIAM J. Appl. Math. 65 (6) (2005) 1981-2004. doi:10.1137/S0036139902413040

[44] L.-M. Imbert-Gérard, B. Després, A generalized plane-wave numerical method for smooth nonconstant coefficients, IMA J. Numer. Anal. 34 (3) (2014) 1072-1103. doi:10.1093/imanum/drt030 